TRANSACTIONS OF THE

AMERICAN MATHEMATICAL SOCIETY

Volume 365, Number 2, February 2013, Pages 885-909

S 0002-9947(2012)05651-0

Article electronically published on July 2, 2012

\title{
THE WITTEN DEFORMATION FOR EVEN DIMENSIONAL CONFORMALLY CONIC MANIFOLDS
}

\author{
URSULA LUDWIG
}

\begin{abstract}
The goal of this article is to generalise the Witten deformation to even dimensional conformally conic manifolds $X$ and a class of functions $f: X \rightarrow \mathbb{R}$ called admissible Morse functions. We get Morse inequalities relating the $\mathrm{L}^{2}$-Betti numbers of $X$ with the number of critical points of the function $f$. Hereby the contribution of a singular point $p$ of $X$ to the Morse inequalities can be expressed in terms of the intersection cohomology of the local Morse data of $f$ at $p$. The definition of an admissible Morse function is inspired by stratified Morse theory as developed by Goresky and MacPherson.
\end{abstract}

\section{INTRODUCTION}

The Witten deformation is a method proposed in [23] by Witten which, given a smooth Morse function $f: M \rightarrow \mathbb{R}$ on a smooth compact Riemannian manifold $M$, leads to an analytical proof of the Morse inequalities. A rigorous account of the analytic proof of the Morse inequalities using semi-classical analysis has been done in [17. The method has been extended in a sequel of papers to the case of manifolds with boundary (see e.g. [8, [16, 15], 18]). The Witten deformation has been used in 2 2 to give an extension of a theorem of Cheeger and Müller on the relation between the Ray-Singer analytic torsion and the Reidemeister torsion.

This article generalises the Witten deformation for conformally conic manifolds $(X, g)$ of even dimension $\operatorname{dim} X=2 \nu$ and a class of functions which are called admissible Morse functions. Conformally conic manifolds generalise Riemannian manifolds with cone-like singularities (see 4]). The results presented here then also hold in particular for spaces with cone-like singularities. The definition of an admissible Morse function introduced in this article is motivated by the nondegeneracy condition of stratified Morse functions in the sense of stratified Morse theory developed by Goresky and MacPherson [13. However note that the settings in [13] and here are slightly different. The spaces considered in [13] are Whitney stratified spaces (embedded in some $\mathbb{R}^{N}$ ) and the non-degeneracy condition for a stratified Morse function is expressed in terms of the generalised tangent spaces for the Whitney stratification. The main reason for us to work on conformally conic manifolds is that for these spaces $\mathrm{L}^{2}$-techniques are well understood, and we thus have good tools for an analytic proof of the Morse inequalities.

In the rest of this introduction we will explain how to adapt Witten's method to the situation described above, state the main results and explain shortly the idea of the proof.

Received by the editors July 29, 2010 and, in revised form, May 25, 2011.

2010 Mathematics Subject Classification. Primary 35A20; Secondary 57R70.

Key words and phrases. Stratified Morse theory, conic singularities, Witten deformation.

The author was supported in part by SFB 647. 
The main principle in Morse theory is to give a relation between a "local datum" of the Morse function, namely its critical points, and a "global topological datum" of the space. For smooth manifolds the latter is the singular cohomology of the manifold. In the presence of singularities the topological invariant of interest is the so-called intersection cohomology.

For conformally conic manifolds intersection cohomology can be analytically expressed by using the complex of $\mathrm{L}^{2}$-forms: Let us denote by $\Sigma$ the singular set of $X$. Let $\left(\Omega_{0}^{*}(X \backslash \Sigma), d\right)$ be the de Rham complex of differential forms with compact supports. For conformally conic manifolds the elliptic complex $\left(\Omega_{0}^{*}(X \backslash \Sigma), d\right)$ admits a unique extension into a Hilbert complex $(\mathcal{C}, d,\langle\rangle$,$) in the Hilbert space of$ square integrable forms equipped with the $\mathrm{L}^{2}$-metric:

$$
\langle\alpha, \beta\rangle:=\int_{X \backslash \Sigma} \alpha \wedge * \beta
$$

The $\mathrm{L}^{2}$-cohomology of $X$, denoted by $H_{(2)}^{i}(X)$, is defined as the cohomology of this Hilbert complex. (See Section 2 for details. Note that in this article the language of Hilbert complexes, as introduced in [3], is used.)

Witten's idea for an analytic proof of the Morse inequalities on a smooth compact manifold consists in the deformation of the de Rham complex by means of a smooth Morse function (see [23], [17]). In the presence of singularities we deform the complex of $\mathrm{L}^{2}$-forms instead. We use an admissible Morse function $f: X \rightarrow \mathbb{R}$ for the deformation (see Definition 2.1). In particular we deform the complex $\left(\Omega_{0}^{*}(X \backslash \Sigma), d\right)$ into

$$
0 \rightarrow \Omega_{0}^{0}(X \backslash \Sigma) \stackrel{d_{t}}{\longrightarrow} \ldots \stackrel{d_{t}}{\longrightarrow} \Omega_{0}^{2 \nu}(X \backslash \Sigma) \rightarrow 0,
$$

where $d_{t}=e^{-f t} d e^{f t}$; here $t \in(0, \infty)$ is the deformation parameter. One can show that the deformed complex also admits a unique extension into a Hilbert complex, which is denoted by $\left(\mathcal{C}_{t}, d_{t},\langle\rangle,\right)$. The map $\omega \rightarrow e^{-t f} \omega$ yields an isomorphism of the two complexes $(\mathcal{C}, d,\langle\rangle$,$) and \left(\mathcal{C}_{t}, d_{t},\langle\rangle,\right)$. Therefore the cohomology of the deformed complex is also isomorphic to the $\mathrm{L}^{2}$-cohomology of $X$, i.e. $H^{i}\left(\mathcal{C}_{t}, d_{t},\langle\rangle,\right) \simeq H_{(2)}^{i}(X)$.

Let us denote by $\delta_{t}$ the adjoint of $d_{t}$ with respect to the $\mathrm{L}^{2}$-metric. The Witten Laplacian is defined as the Laplacian associated to the Hilbert complex $\left(\mathcal{C}_{t}, d_{t},\langle\rangle,\right)$, i.e.

$$
\begin{aligned}
& \Delta_{t}=d_{t} \delta_{t}+\delta_{t} d_{t} \\
& \operatorname{dom}\left(\Delta_{t}\right)=\left\{\omega \mid \omega, d_{t} \omega, \delta_{t} \omega, d_{t} \delta_{t} \omega, \delta_{t} d_{t} \omega \in \mathrm{L}^{2}\left(\Lambda^{*}\left(T^{*}(X \backslash \Sigma)\right)\right)\right\} .
\end{aligned}
$$

Note that in the presence of singularities $\left.\Delta_{t}\right|_{\Omega_{0}^{*}(X \backslash \Sigma)}$ is not an essentially self-adjoint operator and therefore we have to specify the domain of the Witten Laplacian carefully. The Witten Laplacian is a non-negative, self-adjoint operator with discrete spectrum. Hodge theory is still valid for the deformed complex, i.e.

$$
\operatorname{ker}\left(\Delta_{t}^{(i)}\right) \simeq H^{i}\left(\mathcal{C}_{t}, d_{t},\langle,\rangle\right), \quad i=0, \ldots, 2 \nu,
$$

where $\Delta_{t}^{(i)}$ denotes the restriction of $\Delta_{t}$ acting on $i$-forms.

The advantage of the deformed complex $\left(\mathcal{C}_{t}, d_{t},\langle\rangle,\right)$ compared to the initial complex $(\mathcal{C}, d,\langle\rangle$,$) is that the spectrum of the Witten Laplacian has nice properties$ for large parameters $t$. For an admissible Morse function $f$ the restriction $f_{\mid X \backslash \Sigma}$ is 
a Morse function in the smooth sense and we denote by $c_{i}\left(f_{\mid X \backslash \Sigma}\right)$ the number of critical points of $f_{\mid X \backslash \Sigma}$ of index $i$.

Theorem 1.1 (Spectral gap theorem). (1) Let $(X, g)$ be an even dimensional conformally conic Riemannian manifold and let $f: X \rightarrow \mathbb{R}$ be an admissible Morse function. Then there exist constants $C_{1}, C_{2}, C_{3}>0$ and $t_{0}>0$ depending on $X$ and $f$ such that for any $t>t_{0}$,

$$
\operatorname{spec}\left(\Delta_{t}\right) \cap\left(C_{1} e^{-C_{2} t}, C_{3} t\right)=\emptyset .
$$

(2) Let us denote by $\left(\mathcal{S}_{t}, d_{t},\langle\rangle,\right)$ the subcomplex of $\left(\mathcal{C}_{t}, d_{t},\langle\rangle,\right)$ generated by all eigenforms of the Witten Laplacian $\Delta_{t}$ to eigenvalues in $[0,1]$. Then, for $t \geq t_{0}$,

$$
\operatorname{dim} \mathcal{S}_{t}^{i}=c_{i}\left(f_{\mid X \backslash \Sigma}\right)+\sum_{p \in \Sigma} m_{p}^{i}=: c_{i}(f)
$$

where $m_{p}^{i}$ is the contribution of the singular point $p$ to $c_{i}(f)$ explained in more detail below.

The precise definition of the $m_{p}^{i}$ is given in Section 3. While it is rather technical we shortly explain it here also for completeness: For a point $p \in \Sigma$ and $\epsilon>0$ let us denote by $B_{\epsilon}(p)$ the closed $\epsilon$-ball around $p$. Then, for $\epsilon$ small enough, $B_{\epsilon}(p)$ is homeomorphic to the closed cone $L_{p} \times[0,1] / 0 \times L_{p}$. The closed $(2 \nu-1)$-dimensional manifold $L_{p}$ is called the link of the singularity. Let us choose $0<\delta \ll \epsilon$. The local Morse data of $f$ at $p \in \Sigma$ reduce in this case to the normal local Morse data and are defined as the pair of spaces

$$
\left(M_{p}, l_{p}^{-}\right):=\left(B_{\epsilon}(p) \cap f^{-1}([f(p)-\delta, f(p)+\delta]), B_{\epsilon}(p) \cap f^{-1}(f(p)-\delta)\right) .
$$

The pair $\left(M_{p}, l_{p}^{-}\right)$is independent of the choice of $0<\delta \ll \epsilon$. As in stratified Morse theory (see [13], pg. 66) we call $l_{p}^{-}:=B_{\epsilon}(p) \cap f^{-1}(f(p)-\delta)$ the lower half-link. It is not difficult to see that the pair $\left(M_{p}, l_{p}^{-}\right)$is homeomorphic to $\left(B_{\epsilon}(p), l_{p}^{-}\right)$. The contribution of the singular point $p$ to the Morse inequalities is related to the local Morse data as follows:

$$
m_{p}^{i}=\operatorname{dim} I H^{i}\left(M_{p}, l_{p}^{-}\right)=\operatorname{dim} I H^{i}\left(B_{\epsilon}(p), l_{p}^{-}\right),
$$

where $I H^{*}$ denotes the intersection cohomology (with closed support).

As usual the following Morse inequalities follow from the spectral gap theorem and the Hodge theory for the deformed complex (1.3) by a simple algebraic argument.

Corollary 1.2. In the situation of Theorem 1.1 ,

$$
\begin{aligned}
& \sum_{i=0}^{k}(-1)^{k-i} c_{i}(f) \geq \sum_{i=0}^{k}(-1)^{k-i} b_{i}^{(2)}(X), \text { for all } 0 \leq k<2 \nu, \\
& \sum_{i=0}^{2 \nu}(-1)^{i} c_{i}(f)=\sum_{i=0}^{2 \nu}(-1)^{i} b_{i}^{(2)}(X),
\end{aligned}
$$

where $b_{i}^{(2)}(X):=\operatorname{dim} H_{(2)}^{i}(X)$ denote the $\mathrm{L}^{2}$-Betti numbers of $X$. 
Note that a particular example of the situation treated here is the case where $X$ is a complex cone and $f: X \rightarrow \mathbb{R}$ is a stratified Morse function in the sense of 13 . Then the Morse inequalities in (1.7) give back the stratified Morse inequalities in [13.

The key step in the proof of the spectral gap theorem is to construct a local model operator $\boldsymbol{\Delta}_{t, p}$ for the Witten Laplacian near a singular point $p$ of $X$ and to show a local spectral gap theorem for $\boldsymbol{\Delta}_{t, p}$.

In a previous paper 20] (see also [19]) the Witten deformation for singular complex curves $C \subset \mathbb{P}^{n}(\mathbb{C})$ equipped with a stratified Morse function in the sense of the theory in 13 has been discussed. There the local model has a particularly simple form which has been treated by an explicit computation. In the higher dimensional situation the local model is more complicated and the local spectral gap theorem is shown using perturbation techniques for regular singular operators as in 5. A cone construction adapted from [7] (see also [1]) is used to prove the relation to the geometry.

These notes are organised as follows: In Section 2 we shortly recall the basic facts on the $\mathrm{L}^{2}$-cohomology of conformally conic manifolds. We also define the class of admissible Morse functions and explain the Witten deformation in this singular context. The results in Section 2 are a direct generalisation of the curve case and they also hold mutatis mutandis for the odd dimensional case. We give the proofs in some detail however for the convenience of the reader. The main work is done in Section 3 where we define the local model operator $\boldsymbol{\Delta}_{t}$ for the Witten Laplacian near a singular point of $X$ and show the local spectral gap theorem. In Section 4 an outline of the proof of the spectral gap theorem (Theorem 1.1) and the Morse inequalities (Corollary 1.2) is given. Once the local situation near singular points of $X$ is understood the proof of the spectral gap theorem is a direct generalisation of the proof in the smooth case (here we follow the proof in [1], Section 9).

The results presented here have been announced in [21.

\section{The WitTEn DEFORMATION FOR CONFORMALly CONIC MANIFOLDS AND ADMISSIBLE FUNCTIONS}

Let $X$ be a topological space, which moreover is a smooth manifold of dimension $\operatorname{dim} X=2 \nu$ outside a set of isolated singularities $\Sigma:=\left\{p_{1}, \ldots, p_{N}\right\}$. Let $g$ be a Riemannian metric on $X \backslash \Sigma$. We call the pair $(X, g)$ a conformally conic manifold (see [4]) if

- For each $p \in \Sigma$ there exists an open neighbourhood $U_{p}$ in $X$ such that $\left(U_{p} \backslash\{p\}, g_{\mid U_{p} \backslash\{p\}}\right)$ is isometric to $\left((0, \epsilon) \times L_{p}, g(r)\right)$. Hereby $L_{p}$ is a closed manifold of dimension $\operatorname{dim}\left(L_{p}\right)=2 \nu-1=: n$ called the link of the singular point $p$. Moreover $g(r)=h(r)^{2}\left(d r^{2} \oplus r^{2} g_{L_{p}}(r)\right)$, where $g_{L_{p}}(r)$ is a family of metrics on $L_{p}$, smooth in $(0, \epsilon)$ and continuous in $[0, \epsilon)$, and $h \in C^{\infty}((0, \epsilon) \times$ $\left.L_{p}\right)$ satisfies

$$
\sup _{\varphi \in L_{p}}\left|\left(r \partial_{r}\right)^{j}\left(r^{-c} h(r, \varphi)-1\right)\right|=O\left(r^{\delta}\right) \text { as } r \rightarrow 0, \quad j=0,1,
$$

and

$$
\sup _{\varphi \in L_{p}}\left\|h(r, \varphi)^{-1} d_{L_{p}} h(r, \varphi)\right\|_{T_{\varphi}^{*} L, g_{L_{p}}(r)}=O\left(r^{\delta}\right) \text { as } r \rightarrow 0,
$$

for some $\delta>0$ and $c>-1$. 
- If we denote by

$$
g^{0}:=d r^{2}+r^{2} g_{L_{p}}(0), \quad g^{1}:=h^{-2} g=d r^{2}+r^{2} g_{L_{p}}(r)
$$

and by $\omega^{0}, \omega^{1}$ the connection forms of the Levi-Civita connections for $g^{0}$, $g^{1}$, then

$$
\sup _{\varphi \in L_{p}}\left(\left|g^{1}-g^{0}\right|_{(r, \varphi)}^{0}+r\left|\omega^{1}-\omega^{0}\right|_{(r, \varphi)}^{0}\right)=O\left(r^{\delta}\right) \text { as } r \rightarrow 0,
$$

where ${ }^{0}$ refers to the metric $g^{0}$.

We will also assume for the rest of this paper that $X$ is compact. Via the transformation $r \mapsto \frac{1}{1+c} r^{c+1}$ the metric $g$ changes into $\tilde{g}=\tilde{h}^{2}\left(d r^{2}+r^{2} \tilde{g}_{L_{p}}(r)\right)$ which satisfies all estimates above with $c=0$. From now on we will always assume that we are in this latter situation.

Conformally conic Riemannian manifolds generalise Riemannian manifolds with cone-like singularities. In particular a conformally conic manifold is quasi-isometric to a conic one. Important examples of conformally conic manifolds are singular complex curves and complex cones (see 4]).

Let $(X, g)$ be a conformally conic Riemannian manifold. Let $\left(\Omega_{0}^{*}(X \backslash \Sigma), d\right)$ be the de Rham complex of differential forms with compact supports. An ideal boundary condition (see [9], 3]) for the elliptic complex $\left(\Omega_{0}^{*}(X \backslash \Sigma), d\right)$ is a choice of closed extensions $D_{k}$ of $d_{k}$ in the Hilbert space of square integrable $k$-forms, such that $D_{k}\left(\operatorname{dom}\left(D_{k}\right)\right) \subset \operatorname{dom}\left(D_{k+1}\right)$. We then get a Hilbert complex

$$
0 \rightarrow \operatorname{dom}\left(D_{0}\right) \stackrel{D_{0}}{\longrightarrow} \cdots \stackrel{D_{n-1}}{\longrightarrow} \operatorname{dom}\left(D_{n}\right) \rightarrow 0 .
$$

We will abbreviate the term ideal boundary condition by $i b c$ in the sequel. The minimal and maximal extensions of $d$,

$$
d_{\min }:=\bar{d}=\text { closure of } d, d_{\max }:=\delta^{*}=\text { adjoint of the formal adjoint } \delta \text { of } d,
$$

are examples of $i b c$ 's. A priori there may be several distinct $i b c$ 's.

As shown in [9] in the case of manifolds with cone-like singularities we have uniqueness of ideal boundary condition, i.e. in that case the minimal and the maximal extensions coincide. We also say that the $\mathrm{L}^{2}$-Stokes theorem holds for cone-like singularities. The domains of $d_{\min }$ and $d_{\max }$, and therefore the validity of the $\mathrm{L}^{2}$-Stokes theorem, are quasi-isometry invariants. Therefore also in the case of conformally conic manifolds we have a unique $i b c$, i.e.

$$
d_{k, \min }=d_{k, \max } \text { for all } k \text {. }
$$

We denote by $(\mathcal{C}, d,\langle\rangle$,$) the unique extension of the differential complex \left(\Omega_{0}^{*}(X \backslash\right.$ $\Sigma), d)$ to a Hilbert complex. The cohomology of this complex is the so-called $\mathrm{L}^{2}-$ cohomology of $X$ :

$$
H_{(2)}^{i}(X):=\operatorname{ker} d_{i, \min } / \operatorname{im} d_{i-1, \min }=\operatorname{ker} d_{i, \max } / \operatorname{im} d_{i-1, \max } .
$$

Since the $\mathrm{L}^{2}$-cohomology of $X$ is a quasi-isometry invariant one can compute it also by replacing the conformally conic metric with a conic metric. Therefore it is clear that all $\mathrm{L}^{2}$-cohomology groups $H_{(2)}^{i}(X)$ are finite dimensional and the complex $(\mathcal{C}, d,\langle\rangle$,$) is Fredholm. Applying Corollary 2.5$ in 3 . to the Fredholm complex $(\mathcal{C}, d,\langle\rangle$,$) one deduces that the canonical maps$

$$
\operatorname{ker}\left(\Delta^{(i)}\right) \longrightarrow H_{(2)}^{i}(X)
$$


are isomorphisms, where by $\Delta$ we denote the Laplacian associated to the Hilbert complex $(\mathcal{C}, d,\langle\rangle$,$) and by \Delta^{(i)}$ its restriction to $i$-forms.

The uniqueness of $i b c$ in the case of conformally conic manifolds has also been shown by Brüning and Lesch in 4] by a different argument, which will be useful here.

In this section we perform the Witten deformation on the complex of $\mathrm{L}^{2}$-forms by means of a certain class of functions, which we call admissible functions:

Definition 2.1. $\quad$ a) A continuous function $f: X \rightarrow \mathbb{R}$ is called admissible if its restriction to $X \backslash \Sigma$ is smooth and moreover locally near a singularity $p \in \Sigma$ the function $f$ has the form

$$
f(r, \varphi)=f(p)+f_{1}(r, \varphi)+f_{2}(r, \varphi), \text { where } f_{1}=r h, f_{2}=O\left(r^{1+\delta}\right)
$$

and $h: L_{p} \rightarrow \mathbb{R}$ is a smooth function.

b) An admissible function $f: X \rightarrow \mathbb{R}$ is called an admissible Morse function if

(i) the restriction $\left.f\right|_{X \backslash \Sigma}$ is Morse in the smooth sense;

(ii) there exists a neighbourhood $U$ of the singular set $\Sigma$ and a constant $a>0$ such that $|\nabla f|^{2} \geq a^{2}$ on $U$.

Remark 2.2. The condition (ii) in the definition of an admissible Morse function is inspired by stratified Morse theory as developed by Goresky and MacPherson [13]: A stratified Morse function is not critical in normal directions for any critical point on a lower dimensional stratum. It will enter in our analysis in two different ways, which will become more transparent in Section 3: It allows us to show the local spectral gap theorem for the model Witten Laplacian. Moreover we can adapt a method from [7, where the Witten deformation for manifolds with boundary has been studied, to show the geometric content of $m_{p}^{i}$, the contribution of the singular point $p \in \Sigma$ to the Morse inequalities.

For the rest of this section let $f: X \rightarrow \mathbb{R}$ be an admissible function on the conformally conic manifold $X$. Let us denote by $\left(\Omega_{0}^{*}(X \backslash \Sigma), d_{t},\langle\rangle,\right)$ the differential complex of smooth forms with compact supports on $X \backslash \Sigma$, where $d_{t}=e^{-f t} d e^{f t}$ and $\langle$,$\rangle is the \mathrm{L}^{2}$-metric, $t \in(0, \infty)$ is the deformation parameter. We have two interesting associated Hilbert complexes: the maximal extension $\left(\mathcal{C}_{t, \max }, d_{t, \max },\langle\rangle,\right)$, defined by

$$
d_{t, \max }=\text { adjoint of the formal adjoint of } d_{t} \text { w. r. t. }\langle,\rangle,
$$

and the minimal extension $\left(\mathcal{C}_{t, \min }, d_{t, \min },\langle\rangle,\right)$, defined by

$$
d_{t, \min }=\text { closure of } d_{t} \text { with respect to }\langle,\rangle \text {. }
$$

Denote by $\delta_{t}$ the formal adjoint of the operator $d_{t}$ with respect to the metric $\langle$,$\rangle ,$ and by $\left.\Delta_{t}\right|_{\Omega_{0}^{*}}=\left(d_{t}+\delta_{t}\right)^{2}$ the deformed Laplacian (acting on smooth compactly supported forms).

Lemma 2.3. The following identities hold for $\omega \in \Omega_{0}^{*}(X \backslash \Sigma)$ :

$$
\begin{aligned}
d_{t} \omega & =d \omega+t d f \wedge \omega, \\
\delta_{t} \omega & =e^{t f} \delta e^{-t f} \omega=\delta \omega+t \nabla f-\omega, \\
\Delta_{t} \omega & =\Delta \omega+t\left(\mathcal{L}_{\nabla f}+\mathcal{L}_{\nabla f}^{*}\right) \omega+t^{2}|\nabla f|^{2} \omega,
\end{aligned}
$$

where we denote by $\left.\mathcal{L}_{\nabla f}=d(\nabla f\lrcorner\right)+\nabla f \_d$ the Lie derivative in the direction of the gradient vector field $\nabla f$ and by $\mathcal{L}_{\nabla f}^{*}$ its adjoint. 
Proof. See e.g. Prop. 5.4 in [2].

Remark 2.4. Note that the operator $M_{f}:=\mathcal{L}_{\nabla f}+\mathcal{L}_{\nabla f}^{*}$ is a zeroth order operator.

Let us denote by

$$
D_{t}^{\mathrm{ev}}:=d_{t}+\delta_{t}: \Omega_{0}^{\mathrm{ev}}(X \backslash \Sigma) \longrightarrow \Omega_{0}^{\mathrm{odd}}(X \backslash \Sigma)
$$

and by

$$
D_{t}^{\text {odd }}:=d_{t}+\delta_{t}: \Omega_{0}^{\text {odd }}(X \backslash \Sigma) \longrightarrow \Omega_{0}^{\mathrm{ev}}(X \backslash \Sigma) .
$$

The operator $D_{t}:=D_{t}^{\text {ev }}+D_{t}^{\text {odd }}$ is a closable operator with

$\operatorname{dom}\left(D_{t, \min }\right)=\left\{\begin{array}{c}\omega \in \mathrm{L}^{2} \mid \text { there exists a sequence } \phi_{n} \in \Omega_{0}^{*}(X) \text { s.t. } \phi_{n} \rightarrow \omega \\ \text { and } d_{t} \phi_{n}, \delta_{t} \phi_{n} \text { are Cauchy sequences in } \mathrm{L}^{2}\left(\Lambda^{*}\left(T^{*}(X \backslash \Sigma)\right)\right)\end{array}\right\}$.

Thus in particular

$$
\operatorname{dom}\left(D_{t, \min }\right) \subset \operatorname{dom}\left(d_{t, \min }\right) \cap \operatorname{dom}\left(\delta_{t, \min }\right) .
$$

Lemma 2.5. Let $X$ be a conformally conic Riemannian manifold of even dimension $\operatorname{dim} X=2 \nu$.

(a) Then we have

$$
\operatorname{dom}\left(d_{t, k, \text { max }}\right) \cap \operatorname{dom}\left(\delta_{t, k-1, \max }\right) \subset \operatorname{dom}\left(D_{t, \text { min }}\right)
$$

for all $k$, except possibly $k=\nu$.

(b) Moreover, for $k \neq \nu$,

$$
\begin{aligned}
& d_{t, k, \max }=d_{t, k, \min }, \\
& \delta_{t, k-1, \max }=\delta_{t, k-1, \min }, \\
& \Delta_{t}^{\mathcal{F},(k)}=d_{t, k-1, \min } \delta_{t, k-1, \min }+\delta_{t, k, \min } d_{t, k, \min },
\end{aligned}
$$

where $\Delta_{t}^{\mathcal{F}}$ denotes the Friedrichs extension of $\left.\Delta_{t}\right|_{\Omega_{0}^{*}(X \backslash \Sigma)}$.

Proof. (a) It is easy to see using the local form of an admissible function $f$ near the singularities and the formulas in Section 3.3 that $d f \wedge: \mathrm{L}^{2} \rightarrow \mathrm{L}^{2}$ and $\left.\nabla f\right\lrcorner: \mathrm{L}^{2} \rightarrow \mathrm{L}^{2}$ are bounded operators. Therefore we get

$$
\begin{aligned}
& \operatorname{dom}\left(d_{t, k, \text {max}}\right)=\operatorname{dom}\left(d_{k, \text { max }}\right), \\
& \operatorname{dom}\left(\delta_{t, k-1, \max }\right)=\operatorname{dom}\left(\delta_{k-1, \max }\right), \\
& \operatorname{dom}\left(D_{t, \text { min }}\right)=\operatorname{dom}\left(D_{\text {min }}\right),
\end{aligned}
$$

where $D=d+\delta$. By Theorem 2.1 in [4] we have, for $k \neq \nu$,

$$
\operatorname{dom}\left(d_{k, \max }\right) \cap \operatorname{dom}\left(\delta_{k-1, \max }\right) \subset \operatorname{dom}\left(D_{\min }\right) .
$$

The claim now follows from (2.19) and (2.20).

(b) By (a) the assumptions of Lemma 3.3 in [4] are satisfied for the complex $\left(\Omega_{0}^{*}(X \backslash \Sigma), d_{t},\langle\rangle,\right)$. By applying the cited result we get the claim.

Proposition 2.6. $\quad$ (a) The complex $\left(\Omega_{0}^{*}(X \backslash \Sigma), d_{t},\langle\rangle,\right)$ satisfies the $\mathrm{L}^{2}$-Stokes theorem, i.e.

$$
\operatorname{dom}\left(d_{t, \max }\right)=\operatorname{dom}\left(d_{t, \min }\right) .
$$

We denote by $\left(\mathcal{C}_{t}, d_{t},\langle\rangle,\right)$ the unique extension of the complex $\left(\Omega_{0}^{*}(X \backslash\right.$ $\left.\Sigma), d_{t},\langle\rangle,\right)$ into a Hilbert complex. 
(b) The complex $\left(\mathcal{C}_{t}, d_{t},\langle\rangle,\right)$ is a Fredholm complex whose cohomology is isomorphic to $H_{(2)}^{i}(X)$, the $\mathrm{L}^{2}$-cohomology of $X$. Moreover, for $i=0, \ldots, 2 \nu$, the natural maps

$$
\operatorname{ker} \Delta_{t}^{(i)}=\operatorname{ker} d_{t, i} \cap \operatorname{ker} \delta_{t, i-1} \longrightarrow H^{i}\left(\mathcal{C}_{t}, d_{t},\langle,\rangle\right) \simeq H_{(2)}^{i}(X)
$$

are isomorphisms, where $\Delta_{t}$ is the Laplacian associated to the complex $\left(\mathcal{C}_{t}, d_{t},\langle\rangle,\right)$.

(c) The operator $\Delta_{t}$ is a discrete operator and moreover, for $k \neq \nu$,

$$
\Delta_{t}^{(k)}=\Delta_{t}^{\mathcal{F},(k)} .
$$

(d) The Gauss-Bonnet operator associated to the complex $\left(\mathcal{C}_{t}, d_{t},\langle\rangle,\right)$, i.e.

$$
D_{t}^{G B}:=\bigoplus_{k \geq 0}\left(d_{t, 2 k, \min }+\delta_{t, 2 k-1, \max }\right)
$$

is Fredholm and satisfies

$$
D_{t}^{G B}:= \begin{cases}D_{t, \min }^{\mathrm{ev}} & \text { if } \nu \text { odd }, \\ D_{t, \mathrm{max}}^{\mathrm{ev}} & \text { if } \nu \text { even } .\end{cases}
$$

Moreover $\operatorname{ind}\left(D_{t}^{G B}\right)=\chi_{(2)}(X)$, where $\chi_{(2)}(X)$ is the $\mathrm{L}^{2}$-Euler characteristic of $X$.

Remark 2.7. We call the Laplacian $\Delta_{t}$ associated to the Hilbert complex $\left(\mathcal{C}_{t}, d_{t},\langle\rangle,\right)$ the Witten Laplacian. By definition (of the Laplacian associated to a Hilbert complex) it is the closed self-adjoint non-negative extension of $\left.\Delta_{t}\right|_{\Omega_{0}^{*}(X \backslash \Sigma)}$ with domain:

$$
\operatorname{dom}\left(\Delta_{t}\right)=\left\{\omega \mid \omega, d_{t} \omega, \delta_{t} \omega, d_{t} \delta_{t} \omega, \delta_{t} d_{t} \omega \in \mathrm{L}^{2}\left(\Lambda^{*}\left(T^{*}(X \backslash \Sigma)\right)\right)\right\} .
$$

Proof. a) Let us denote by $\langle,\rangle_{t}$ the twisted $\mathrm{L}^{2}$-metric:

$$
\langle\alpha, \beta\rangle_{t}=\int_{X \backslash \Sigma} \alpha \wedge * \beta e^{-2 t f} .
$$

Since $f$ is bounded on $X$ the two metrics $\langle$,$\rangle and \langle,\rangle_{t}$ are equivalent. We introduce the following auxiliary differential complex:

$$
\left(\Omega_{0}^{*}(X \backslash \Sigma), \widetilde{d}_{t},\langle,\rangle_{t}\right),
$$

where $\langle,\rangle_{t}$ is the twisted metric defined above and $\widetilde{d}_{t}:=d$ is the usual differential. Using the invariance of the validity of the $\mathrm{L}^{2}$-Stokes theorem for equivalent metrics one deduces easily that the complex $\left(\Omega_{0}^{*}(X \backslash \Sigma), d,\langle,\rangle_{t}\right)$ admits a unique extension to a Hilbert complex, which we denote by

$$
\left(\widetilde{\mathcal{C}}_{t}, \widetilde{d}_{t},\langle,\rangle_{t}\right)=\left(\widetilde{\mathcal{C}}_{t \text { max }}, \widetilde{d}_{t \max },\langle,\rangle_{t}\right)=\left(\widetilde{\mathcal{C}}_{t \text { min }}, \widetilde{d}_{t \text { min }},\langle,\rangle_{t}\right) .
$$

Since $d_{t}\left(e^{-t f} \omega\right)=e^{-t f}\left(\widetilde{d}_{t} \omega\right)$ the map

$$
e^{-t f}:\left(\Omega_{0}^{*}(X \backslash \Sigma), \widetilde{d}_{t},\langle,\rangle_{t}\right) \rightarrow\left(\Omega_{0}^{*}(X \backslash \Sigma), d_{t},\langle,\rangle\right), \quad \omega \mapsto e^{-t f} \omega
$$

is an isomorphism of complexes. It is not difficult to see that the map (2.29) extends to isomorphisms of Hilbert complexes

$$
\left(e^{-t f}\right)_{\max / \min }:\left(\widetilde{\mathcal{C}}_{t_{\max } / \min }, \tilde{d}_{t_{\max } / \min },\langle,\rangle_{t}\right) \simeq\left(\mathcal{C}_{t_{\max } / \min }, d_{t_{\max } / \min },\langle,\rangle\right) .
$$

The claim now follows from the validity of the $\mathrm{L}^{2}$-Stokes theorem for the complex $\left(\widetilde{\mathcal{C}_{t}}, \widetilde{d}_{t},\langle,\rangle_{t}\right)$. 
Alternatively we can prove the claim in a) also by the following argument: By Lemma 2.5 (b) one has

$$
d_{t, k, \max }=d_{t, k, \min }, \delta_{t, k-1, \max }=\delta_{t, k-1, \min } \text { for all } k \neq \nu .
$$

Therefore, since $\delta_{t, k, \min / \max }$ is the adjoint of $d_{t, k, \max / \min }$, we get $d_{t, k, \max }=d_{t, k, \min }$ for all $k$.

(b) Since $(\mathcal{C}, d,\langle\rangle$,$) is Fredholm, \left(\widetilde{\mathcal{C}}_{t}, \widetilde{d}_{t},\langle,\rangle_{t}\right)$ is also Fredholm. Thus by the isomorphism constructed in (a) $\left(\mathcal{C}_{t}, d_{t},\langle\rangle,\right)$ is also Fredholm. The rest of the claim follows using the isomorphism in (a) and general statements for Hilbert complexes in 3] (Theorem 2.4 and Corollary 2.5).

(c) We know already that $\Delta$ is discrete. Since the discreteness of the Laplacian associated to a Hilbert complex is invariant under complex isomorphism (see Lemma 2.17 in [3]), $\Delta_{t}$ is also discrete. The second claim follows from Lemma 2.5]

(d) As for the complex of $\mathrm{L}^{2}$-forms (compare [4, Theorem 3.7 (c)) the closed extensions of $d_{t}+\delta_{t}$ are restricted by the relation

$$
\operatorname{dom}\left(\left(d_{t}+\delta_{t}\right)_{\min }\right) \cap \mathrm{L}^{2}\left(\Lambda^{k} T^{*} X\right)=\operatorname{dom}\left(d_{t, k, \min }\right) \cap \operatorname{dom}\left(\delta_{t, k-1, \min }\right), \quad k \neq \nu
$$

and

$$
p_{k} \operatorname{dom}\left(\left(d_{t}+\delta_{t}\right)_{\max }\right)=\operatorname{dom}\left(d_{t, k, \min }\right) \cap \operatorname{dom}\left(\delta_{t, k-1, \min }\right), \quad|k-\nu| \neq 1,
$$

where $p_{k}$ denotes the orthogonal projection $p_{k}: \mathrm{L}^{2}\left(\Lambda^{*} T^{*}(X \backslash \Sigma)\right) \rightarrow \mathrm{L}^{2}\left(\Lambda^{k} T^{*}(X \backslash\right.$ $\Sigma)$ ). Thus (2.25) follows from part (a), (2.24), (2.32) and (2.33). The Fredholm property of $D_{t}^{G B}$ is equivalent to the Fredholm property of the complex $\left(\mathcal{C}_{t}, d_{t},\langle\rangle,\right)$ (see Theorem 2.4 in [3]). The last statement in (d) is now obvious.

Let $\Delta^{\mathcal{F}}$ denote the Friedrichs extension of $\left.\Delta\right|_{\Omega_{0}^{*}(X \backslash \Sigma)}$. As a corollary we get

Corollary 2.8. (a) The form domains of $\Delta^{\mathcal{F}}$ and $\Delta_{t}^{\mathcal{F}}$ coincide.

(b) For $k \neq \nu$ the form domain of $\Delta^{(k)}$ and the form domain of the Witten Laplacian $\Delta_{t}^{(k)}$ coincide.

Proof. (a) The form domain of $\Delta^{\mathcal{F}}$ is the closure of $\Omega_{0}^{*}(X \backslash \Sigma)$ under the norm

$$
\|\omega\|_{1}^{2}:=\|d \omega\|^{2}+\|\delta \omega\|^{2}+\|\omega\|^{2} .
$$

The form domain of $\Delta_{t}^{\mathcal{F}}$ is defined similarly. Note moreover that for $\omega \in \Omega_{0}^{*}(X \backslash \Sigma)$,

$$
\begin{aligned}
\langle\Delta \omega, \omega\rangle & =\|d \omega\|^{2}+\|\delta \omega\|^{2} \\
& \leq 2\left(\left\|d_{t} \omega\right\|^{2}+\left\|\delta_{t} \omega\right\|^{2}+t^{2}\left\langle|\nabla f|^{2} \omega, \omega\right\rangle\right) \\
& =2\left(\left\langle\Delta_{t} \omega, \omega\right\rangle+t^{2}\left\langle|\nabla f|^{2} \omega, \omega\right\rangle\right) .
\end{aligned}
$$

Similarly

$$
\left\langle\Delta_{t} \omega, \omega\right\rangle \leq 2\left(\langle\Delta \omega, \omega\rangle+t^{2}\left\langle|\nabla f|^{2} \omega, \omega\right\rangle\right) .
$$

The claim in (a) now follows easily since $|\nabla f|^{2}$ is bounded on $X$. The claim in (b) is a consequence of Part (a), Proposition 2.6 (c) and the fact that $\operatorname{dom}\left(\Delta^{(k)}\right)=$ $\operatorname{dom}\left(\Delta^{\mathcal{F},(k)}\right), k \neq \nu$ (see Theorem 3.7 (b) in [4]). 


\section{THE LOCAL MODEL}

From now on we will assume that $f: X \rightarrow \mathbb{R}$ is an admissible Morse function, as in Definition 2.1 (b).

In this section we define a local model operator for the Witten Laplacian near a singular point $p \in \Sigma$. Let us recall that a sufficiently small neighbourhood $U_{p}$ of $p$ is homeomorphic to cone $\left(L_{p}\right)$, where $L_{p}$ is the link of the singularity. In the following we denote by

$$
\operatorname{cone}\left(L_{p}\right):=[0, \infty) \times L_{p} /\{0\} \times L_{p}
$$

the infinite cone over $L_{p}$. For $\epsilon>0$ we denote by cone $_{\epsilon}(L)$ the truncated open cone

$$
\operatorname{cone}_{\epsilon}(L):=\{(r, \varphi) \in \operatorname{cone}(L) \mid r<\epsilon\} .
$$

3.1. The local Morse data. Let $B_{\epsilon}(p)$ be the closed $\epsilon$-ball around $p \in \Sigma$ in $X$. Let us choose $0<\delta \ll \epsilon$. Since $p$ is an isolated singular point of $X$ the local Morse data of $f$ at $p \in \Sigma$ reduces in this case to the normal local Morse data. They are defined as the pair of spaces

$$
\left(M_{p}, l_{p}^{-}\right):=\left(B_{\epsilon}(p) \cap f^{-1}([f(p)-\delta, f(p)+\delta]), B_{\epsilon}(p) \cap f^{-1}(f(p)-\delta)\right) .
$$

The local Morse data are independent of the choice of $0<\delta \ll \epsilon$ small enough. The set

$$
l_{p}^{-}:=B_{\epsilon}(p) \cap f^{-1}(f(p)-\delta)
$$

can be seen as an "exit set" for the negative gradient flow. As in stratified Morse theory (see [13, pg. 66) we will call $l_{p}^{-}$the lower half-link of $f$ at $p$. It is not difficult to see that the pair $\left(M_{p}, l_{p}^{-}\right)$is homeomorphic to $\left(B_{\epsilon}(p), l_{p}^{-}\right)$and thus

$$
I H^{*}\left(M_{p}, l_{p}^{-}\right) \simeq I H^{*}\left(\operatorname{cone}\left(L_{p}\right), l_{p}^{-}\right),
$$

where $I H^{*}$ denotes intersection cohomology with closed support.

For simplicity we will write from now on $L, l^{-}$, etc. instead of $L_{p}, l_{p}^{-}$, etc. in this section. We will also assume that $f(p)=0$.

3.2. Definition of the model operator. Local spectral gap theorem. Let us fix $\epsilon>0$. Let $\eta_{\epsilon}: \operatorname{cone}(L) \rightarrow[0,1]$ be a smooth cutoff function, with $\left.\eta_{\epsilon}\right|_{\text {cone }_{\epsilon}(L)} \equiv 1$ and $\left.\eta_{\epsilon}\right|_{\text {cone }(L) \backslash \operatorname{cone}_{2 \epsilon}(L)} \equiv 0$.

We denote by (cone $\left.(L), g_{\text {model }}\right)$ the infinite cone over $L$ equipped with the metric

$$
g_{\text {model }}=\eta_{\epsilon} g+\left(1-\eta_{\epsilon}\right) g_{\text {cone }}, \text { where } g_{\text {cone }}=d r^{2}+r^{2} g_{L}(0) \text {. }
$$

Let $f: \operatorname{cone}(L) \rightarrow \mathbb{R}$ be a function on the infinite cone such that:

$$
f=\eta_{\epsilon}\left(f_{1}+f_{2}\right)+\left(1-\eta_{\epsilon}\right) f_{1},
$$

where $f_{1}=r h, f_{2}=O\left(r^{1+\delta}\right),|\nabla f|^{2} \geq a^{2}>0$.

Let $\langle\rangle:,=\langle,\rangle_{\text {model }}$ be the $\mathrm{L}^{2}$-metric on forms induced by $g_{\text {model }}$. Let $\left(\Omega_{0}^{*}(\operatorname{cone}(L)), d,\langle\rangle,\right)$ be the de Rham complex of smooth compactly supported forms on the infinite cone $\left(\operatorname{cone}(L), g_{\text {model }}\right)$. We denote by $\left(\Omega_{0}^{*}(\operatorname{cone}(L)), d_{t},\langle\rangle,\right)$ the complex obtained by deforming the de Rham complex by means of the function $f$, i.e. $d_{t}:=e^{-t f} d e^{t f}$. 
Theorem 3.1.

(a) There is a unique Hilbert complex $\left(\mathcal{D}_{t}, d_{t},\langle\rangle,\right)$ extending the complex

$$
\left(\Omega_{0}^{*}(\operatorname{cone}(L)), d_{t},\langle,\rangle\right) .
$$

(b) The complex $\left(\mathcal{D}_{t}, d_{t},\langle\rangle,\right)$ is Fredholm. The natural maps

$$
\operatorname{ker}\left(\boldsymbol{\Delta}_{t}^{(i)}\right) \rightarrow H^{i}\left(\mathcal{D}_{t}, d_{t},\langle,\rangle\right), \quad i=0, \ldots, 2 \nu,
$$

are isomorphisms, where $\boldsymbol{\Delta}_{t}$ denotes the Laplacian associated to the Hilbert complex $\left(\mathcal{D}_{t}, d_{t},\langle\rangle,\right)$ and $\boldsymbol{\Delta}_{t}^{(i)}$ its restriction to $i$-forms.

(c) The model Witten Laplacian $\boldsymbol{\Delta}_{t}$ satisfies a local spectral gap theorem: there exists $c>0$ such that for $t$ large enough,

$$
\operatorname{spec}\left(\boldsymbol{\Delta}_{t}^{(i)}\right) \subset\{0\} \cup\left[c t^{2}, \infty\right) .
$$

Moreover all forms in $\operatorname{ker}\left(\boldsymbol{\Delta}_{t}\right)$, as well as their derivatives, have exponential decay outside a small neighbourhood of the singularity.

(d) For the cohomology of the complex $\left(\mathcal{D}_{t}, d_{t},\langle\rangle,\right)$ one gets

$$
H^{i}\left(\mathcal{D}_{t}, d_{t},\langle,\rangle\right) \simeq I H^{i}\left(\operatorname{cone}(L), l^{-}\right) .
$$

Remark 3.2. We call the operator $\boldsymbol{\Delta}_{t}$ defined in Theorem (3.1) (b) the model Witten Laplacian. By definition (of the Laplacian associated to a Hilbert complex), the Laplacian $\boldsymbol{\Delta}_{t}$ associated to the complex $\left(\mathcal{D}_{t}, d_{t},\langle\rangle,\right)$ has domain

$$
\operatorname{dom}\left(\boldsymbol{\Delta}_{t}\right)=\left\{\omega \mid \omega, d_{t} \omega, \delta_{t} \omega, d_{t} \delta_{t} \omega, \delta_{t} d_{t} \omega \in \mathrm{L}^{2}\left(\Lambda^{*}(\operatorname{cone}(L))\right)\right\} .
$$

For simplicity of presentation we prove Theorem 3.1 only in the case of the infinite cone (cone $\left.(L), g_{\text {cone }}=d r^{2}+r^{2} g_{L}(0)\right)$ equipped with the conic metric and the function $f=r h$. The perturbation argument needed to extend the proof to the case of a conformally conic metric and the more general function $f=r h+O\left(r^{1+\delta}\right)$ are detailed in [19] for the case of a complex curve and can easily be adapted to the situation here.

3.3. A useful unitary transformation. From now on in this section we always treat the model case $\left(\right.$ cone $\left.(L), g_{\text {cone }}=d r^{2}+r^{2} g_{L}(0)\right), f=r h$.

Let us denote by $n:=\operatorname{dim} L=2 \nu-1$. We denote by $\pi$ the projection $\pi$ : $L \times \mathbb{R}^{+} \rightarrow L$ and following [6] we define bijective maps

$$
\begin{aligned}
U_{k}: C_{0}^{\infty}\left(\mathbb{R}^{+}, \Omega^{k-1}(L) \oplus \Omega^{k}(L)\right) & \longrightarrow \Omega_{0}^{k}(\operatorname{cone}(L)), \\
\left(\phi_{k-1}, \phi_{k}\right) & \mapsto r^{k-1-n / 2} \pi^{*} \phi_{k-1} \wedge d r+r^{k-n / 2} \pi^{*} \phi_{k},
\end{aligned}
$$

which extend to unitary maps

$$
U_{k}: \mathrm{L}^{2}\left(\mathbb{R}^{+}, \mathrm{L}^{2}\left(\Lambda^{k-1} T^{*} L \oplus \Lambda^{k} T^{*} L, g_{L}(0)\right)\right) \rightarrow \mathrm{L}^{2}\left(\Lambda^{k} T^{*}(\operatorname{cone}(L))\right),
$$

$k=0, \ldots, n+1$.

Let $\Omega^{\mathrm{ev}}, \Omega^{\text {odd }}$ denote even and odd forms, respectively. The $U_{k}$ 's induce unitary maps

$$
\begin{aligned}
& U_{\mathrm{ev}} \quad: \quad C_{0}^{\infty}\left(\mathbb{R}_{+}, \Omega^{*}(L)\right) \longrightarrow \Omega_{0}^{\mathrm{ev}}(\operatorname{cone}(L)), \\
& \left(\phi_{0}, \ldots, \phi_{n}\right) \quad \mapsto \quad\left(U_{0}\left(0, \phi_{0}\right), U_{2}\left(\phi_{1}, \phi_{2}\right), \ldots, U_{n+1}\left(\phi_{n}, 0\right)\right), \\
& U_{\text {odd }}: C_{0}^{\infty}\left(\mathbb{R}_{+}, \Omega^{*}(L)\right) \quad \longrightarrow \quad \Omega_{0}^{\text {odd }}(\operatorname{cone}(L)) \text {, } \\
& \left(\phi_{0}, \ldots, \phi_{n}\right) \quad \mapsto \quad\left(U_{1}\left(\phi_{0}, \phi_{1}\right), U_{3}\left(\phi_{2}, \phi_{3}\right), \ldots, U_{n}\left(\phi_{n-1}, \phi_{n}\right)\right) \text {. }
\end{aligned}
$$


We denote by $\mathbf{D}^{\mathrm{ev}}$ the following operator acting on even forms:

$$
\mathbf{D}^{\mathrm{ev}}:=d+\delta: \Omega_{0}^{\mathrm{ev}}(\operatorname{cone}(L)) \rightarrow \Omega_{0}^{\mathrm{odd}}(\operatorname{cone}(L)) .
$$

In the following, operators on the link are labeled with . An easy computation (see [6], Section 5) shows that

$$
U_{\text {odd }}^{-1} \mathbf{D}^{\mathrm{ev}} U_{\mathrm{ev}}=\partial_{r}+r^{-1} S_{0},
$$

where $S_{0}$ is the operator

$$
S_{0}:=\left(\begin{array}{cccccc}
c_{0} & \tilde{\delta} & & & & \\
\tilde{d} & c_{1} & \tilde{\delta} & & & \\
& \tilde{d} & c_{2} & \tilde{\delta} & & \\
& & \ddots & \ddots & \ddots & \\
& & & \tilde{d} & c_{n-1} & \tilde{\delta} \\
& & & & \tilde{d} & c_{n}
\end{array}\right), \quad c_{i}:=(-1)^{i}\left(i-\frac{n}{2}\right) .
$$

Similarly for $\mathbf{D}^{\text {odd }}:=d+\delta: \Omega_{0}^{\text {odd }}(\operatorname{cone}(L)) \rightarrow \Omega_{0}^{\mathrm{ev}}(\operatorname{cone}(L))$ we get

$$
U_{\text {ev }}^{-1} \mathbf{D}^{\text {odd }} U_{\text {odd }}=-\partial_{r}+r^{-1} S_{0} .
$$

For the Laplacian $\boldsymbol{\Delta}^{\text {ev/odd }}$ acting on even, resp. odd, forms on the infinite cone we then get:

$$
\begin{aligned}
& \mathbb{T}^{\mathrm{ev}}:=U_{\text {ev }}^{-1} \boldsymbol{\Delta}^{\mathrm{ev}} U_{\mathrm{ev}}=-\partial_{r}^{2}+r^{-2}\left(S_{0}^{2}+S_{0}\right), \\
& \mathbb{T}^{\text {odd }}:=U_{\text {odd }}^{-1} \boldsymbol{\Delta}^{\text {odd }} U_{\text {odd }}=-\partial_{r}^{2}+r^{-2}\left(S_{0}^{2}-S_{0}\right) .
\end{aligned}
$$

For the operators

$$
\begin{aligned}
& \mathbf{D}_{t}^{\text {ev }}:=d_{t}+\delta_{t}: \Omega_{0}^{\text {ev }}(\operatorname{cone}(L)) \rightarrow \Omega_{0}^{\text {odd }}(\operatorname{cone}(L)), \\
& \mathbf{D}_{t}^{\text {odd }}:=d_{t}+\delta_{t}: \Omega_{0}^{\text {odd }}(\operatorname{cone}(L)) \rightarrow \Omega_{0}^{\mathrm{ev}}(\operatorname{cone}(L))
\end{aligned}
$$

associated to the deformed complex, one computes easily that

$$
\begin{aligned}
& U_{\text {odd }}^{-1} \mathbf{D}_{t}^{\text {ev }} U_{\text {ev }}=\partial_{r}+r^{-1} S_{0}+t T, \\
& U_{\text {ev }}^{-1} \mathbf{D}_{t}^{\text {odd }} U_{\text {odd }}=-\partial_{r}+r^{-1} S_{0}+t T,
\end{aligned}
$$

where $T$ is the operator

$$
T=\left(\begin{array}{cccccc}
(-1)^{0} h & \widetilde{\nabla} h\lrcorner & & & & \\
\widetilde{d} h & (-1) h & \widetilde{\nabla} h\lrcorner & & & \\
& \widetilde{d} h & (-1)^{2} h & \widetilde{\nabla} h\lrcorner & & \\
& & \ddots & \ddots & \ddots & \\
& & & \widetilde{d} h & (-1)^{n-1} h & \widetilde{\nabla} h\lrcorner \\
& & & & \widetilde{d} h & (-1)^{n} h
\end{array}\right)
$$

For the model Witten Laplacian acting on even/odd forms one gets:

$$
\mathbb{T}_{t}^{\text {ev/odd }}:=U_{\text {ev } / \text { odd }}^{-1} \Delta_{t}^{\text {ev/odd }} U_{\text {ev } / \text { odd }}=\mathbb{T}^{\text {ev/odd }}+t r^{-1} M_{h}+t^{2} T^{2}
$$


where

$(3.23)$

$$
\begin{aligned}
M_{h}:=\left(T S_{0}+S_{0} T\right) \\
=\left(\begin{array}{lll}
\widetilde{\mathcal{L}_{\nabla h}}+\widetilde{\mathcal{L}_{\nabla h}^{*}}+2(-1)^{0} c_{0} h & \\
& \ddots & \widetilde{\mathcal{L}_{\nabla h}}+\widetilde{\mathcal{L}_{\nabla h}^{*}}+2(-1)^{n-1} c_{n-1} h
\end{array}\right)
\end{aligned}
$$

and

$$
T^{2}=|\nabla f|^{2} \cdot \mathrm{Id}=\left(h^{2}+|\widetilde{\nabla} h|^{2}\right) \cdot \mathrm{Id} .
$$

In the sequel the following rescaling argument will be useful:

Lemma 3.3. For $t>0$ denote by $R_{t}$ the unitary rescaling operator

$$
R_{t}(f(r, \varphi))=\sqrt{t} f(t r, \varphi) .
$$

Then

$$
\boldsymbol{\Delta}_{t}=t^{2} R_{t} \boldsymbol{\Delta}_{1} R_{t}^{-1}
$$

Proof. The claim follows by an easy computation.

3.4. Proof of Theorem 3.1 (a). The proof is similar to that of Proposition 2.6 (a).

3.5. Proof of Theorem 3.1 (b). Recall that by Definition 2.1 of an admissible Morse function there exists a constant $a>0$ such that

$$
|\nabla f|^{2}=h^{2}+|\widetilde{\nabla} h|^{2} \geq a^{2} .
$$

Moreover by (3.23) $M_{h}$ is a bounded zeroth order operator on the link $L$, i.e. there exists $C>0$ such that

$$
\left\|M_{h}\right\| \leq C \text {. }
$$

Let us fix $\delta>0$ arbitrarily small, $0<\delta \ll \epsilon$. Let $\eta_{1}$ : cone $(L) \rightarrow[0,1]$ be a cutoff function with supp $\eta_{1} \subset \operatorname{cone}_{\delta}(L)$ and depending only on the radial coordinate, $\eta_{1}(r, \varphi)=\eta_{1}(r)$. Let us denote by

$$
\tau:=\mathbb{T}+2 r^{-1}\left(S_{0} \tilde{T}+\tilde{T} S_{0}\right), \text { where } \tilde{T}=\eta_{1} T .
$$

Let us denote by \|\|$_{\tau}$, resp. \|\|$_{\mathbb{T}}$, the two norms

$$
\begin{aligned}
\|u\|_{\tau}^{2} & :=(\tau u, u)+\|u\|^{2}, \\
\|u\|_{\mathbb{T}}^{2} & :=(\mathbb{T} u, u)+\|u\|^{2} .
\end{aligned}
$$

The operator $\pm \partial_{r}+r^{-1}\left(S_{0}+r \tilde{T}\right)$ will be treated as a perturbation of the operator $\pm \partial_{r}+r^{-1} S_{0}$. We apply the techniques in [5], Section 3 to handle the situation.

Lemma 3.4. $\quad$ (a) The operator $\tau$ is a perturbation of $\mathbb{T}$ which can be written as

$$
\tau=\mathbb{T}+\sum_{i, j} \Phi_{j}^{*} C_{i j} \Phi_{i}
$$


where $C_{i j}$ are operator-valued functions with support in $\operatorname{cone}_{\delta}(L)$ and

$$
\max _{i j}\left\|C_{i j}\right\|=: \widetilde{\epsilon}(\delta)
$$

can be made arbitrarily small by choosing $\delta$ small enough. Moreover the operators $\Phi_{i}$ are controlled by $\mathbb{T}$, i.e. there exists $c_{i}>0$ such that

$$
\left\|\Phi_{i} u\right\|^{2} \leq c_{i}\|u\|_{\mathbb{T}}^{2} \text { for } u \in C_{0}^{\infty}\left(\mathbb{R}_{+}, \Omega^{*-1}(L) \oplus \Omega^{*}(L)\right) .
$$

(b) There exists $c>0$ such that for $u \in C_{0}^{\infty}\left(\mathbb{R}_{+}, \Omega^{*-1}(L) \oplus \Omega^{*}(L)\right)$,

$$
(\tau u, u) \geq-c \widetilde{\epsilon}(\delta)\|u\|^{2}=:-\epsilon(\delta)\|u\|^{2} .
$$

Proof. (a) A computation similar to that in [5] shows the claim. (b) As a consequence of (a) one has

$$
\left|\|u\|_{\tau}^{2}-\|u\|_{\mathbb{T}}^{2}\right| \leq c \widetilde{\epsilon}(\delta)\|u\|_{\mathbb{T}}^{2} \text { for } u \in C_{0}^{\infty}\left(\mathbb{R}_{+}, \Omega^{*-1}(L) \oplus \Omega^{*}(L)\right)
$$

for an appropriate constant $c>0$. Hence

$$
\langle\tau u, u\rangle \geq(1-c \widetilde{\epsilon}(\delta))\langle\mathbb{T} u, u\rangle-c \widetilde{\epsilon}(\delta)\|u\|^{2} \geq-c \widetilde{\epsilon}(\delta)\|u\|^{2},
$$

since $\langle\mathbb{T} u, u\rangle \geq 0$ for $u \in C_{0}^{\infty}\left(\mathbb{R}_{+}, \Omega^{*-1}(L) \oplus \Omega^{*}(L)\right)$.

Using the boundedness of $M_{h}$ (see (3.28) ) there exists $\gamma>0$ such that for any cutoff function $\eta_{2}: \operatorname{cone}(L) \rightarrow[0,1], \operatorname{supp}\left(\eta_{2}\right) \subset \operatorname{cone}(L) \backslash \operatorname{cone}_{\gamma}(L),\left.\eta_{2}\right|_{\text {cone }(L) \backslash \operatorname{cone}_{2 \gamma}(L)}$ $\equiv 1$ we have

$$
\left|\left\langle\eta_{2} r^{-1} M_{h} u, u\right\rangle\right| \leq \epsilon(\delta)\|u\|^{2} .
$$

We write the term $r^{-1} M_{h}$ as a sum

$$
r^{-1} M_{h}=V_{1}+V_{2}+V_{3},
$$

where $V_{1}:=r^{-1}\left(S_{0} \tilde{T}+\tilde{T} S_{0}\right)$ and $V_{2}:=\eta_{2} r^{-1} M_{h}$. Thus the complement $V_{3}$ is a compactly supported potential, whose support does not contain the singularity.

Recall that $\mathbb{T}_{1}$ is the operator defined in (3.18) with $t=1$. We write the operator $\mathbb{T}_{1}$ as a sum of two operators, namely $\mathbb{T}_{1}=: K+L$, where

$$
K:=\frac{1}{2} \tau+V_{2}+\left(|\nabla f|^{2}-a^{2}\right), \quad L:=\frac{1}{2} \mathbb{T}+V_{3}+a^{2} .
$$

We denote by $\mathbb{T}^{\mathcal{F}}$ (resp. $\left.\mathbb{T}_{1}^{\mathcal{F}}\right)$ the Friedrichs extension of $\left.\mathbb{T}\right|_{C_{0}^{\infty}\left(\mathbb{R}_{+}, \Omega^{*-1}(L) \oplus \Omega^{*}(L)\right)}$ (resp. $\left.\left.\mathbb{T}_{1}\right|_{C_{0}^{\infty}\left(\mathbb{R}_{+}, \Omega^{*-1}(L) \oplus \Omega^{*}(L)\right)}\right)$.

Lemma 3.5. (a) We have $K \geq-\frac{3}{2} \epsilon(\delta)$ on $C_{0}^{\infty}\left(\mathbb{R}_{+}, \Omega^{*-1}(L) \oplus \Omega^{*}(L)\right)$.

(b) The operator $L$ in (3.39) with $\operatorname{dom}(L)=\operatorname{dom}\left(\mathbb{T}^{\mathcal{F}}\right)$ is bounded from below and $\operatorname{spec}_{\mathrm{ess}}(L) \subset\left[a^{2}, \infty\right)$.

(c) We have $\operatorname{spec}_{\text {ess }}\left(\mathbb{T}_{1}^{\mathcal{F}}\right) \subset\left[\frac{a^{2}}{2}, \infty\right)$.

Proof. (a) The claim follows using (3.34), (3.37) and (3.27). (b) The first claim is obvious from the definition of $L$, the fact that $V_{3}$ has compact support and $\mathbb{T}^{\mathcal{F}} \geq 0$. We show next that, since $V_{3}$ is a continuous compactly supported potential, it is relatively compact with respect to $\left(\frac{1}{2} \mathbb{T}^{\mathcal{F}}+a^{2}\right)$. We have to show that every sequence $\phi_{n}$ such that $\left\|\left(\frac{1}{2} \mathbb{T}^{\mathcal{F}}+a^{2}\right) \phi_{n}\right\|^{2}+\left\|\phi_{n}\right\|^{2}$ is bounded has a subsequence $\phi_{n_{k}}$ such that $V_{3} \phi_{n_{k}}$ is convergent (in $\mathrm{L}^{2}$ ). From the boundedness of $\left\|\left(\frac{1}{2} \mathbb{T}^{\mathcal{F}}+a^{2}\right) \phi_{n}\right\|^{2}+\left\|\phi_{n}\right\|^{2}$ one gets the boundedness of $\left\|\phi_{n}\right\|_{H^{1}(\Omega)}$ for every bounded domain $\Omega, \operatorname{supp}\left(V_{3}\right) \subset \operatorname{int}(\Omega), 0 \notin \Omega$. By Rellich's compactness theorem 
there exists a subsequence $\phi_{n_{k}}$ such that $\left.\phi_{n_{k}}\right|_{\Omega}$ is convergent in $\mathrm{L}^{2}(\Omega)$. This implies the $\mathrm{L}^{2}$-convergence of $V_{3} \phi_{n_{k}}$.

We can now apply Weyl's theorem (see e.g. 22, XIII.4, Corollary 2) and get

$$
\operatorname{spec}_{\text {ess }}(L)=\operatorname{spec}_{\text {ess }}\left(\frac{1}{2} \mathbb{T}^{\mathcal{F}}+a^{2}\right) \subset\left[a^{2}, \infty\right) .
$$

(c) Note first that, similarly to Corollary 2.8 one can show that the form domains of $\mathbb{T}^{\mathcal{F}}$ and $\mathbb{T}_{1}^{\mathcal{F}}$ coincide. From (a) and (b) we deduce using the min-max principle (for the associated quadratic forms; see e.g. [22, Theorem XIII.2) that $\operatorname{spec}_{\text {ess }}\left(\mathbb{T}_{1}^{\mathcal{F}}\right) \subset$ $\left[a^{2}-\frac{3}{2} \epsilon(\delta), \infty\right)$ and we get the claim by choosing $\epsilon(\delta)$ small enough.

With the tools provided in Lemma 3.4 and Lemma 3.5 we can prove the local spectral gap theorem in all degrees $k \neq \nu$. To prove the result also in middle degree $\nu$, we will use a "complex argument". We formulate the argument in full generality in the language of Hilbert complexes introduced in [3]: Let $(\mathcal{C}, d,\langle\rangle$, be a Hilbert complex. We denote by $\mathcal{D}_{i}$ (resp. $\mathcal{R}_{i}$ ) the domain (resp. the range) of $d_{i}$. By definition of a Hilbert complex the operators $d_{i}$ are closed and densely defined operators in some Hilbert space $H_{i}$ and moreover $\mathcal{R}_{i} \subset \mathcal{D}_{i+1}$. Thus we have a complex

$$
0 \rightarrow \mathcal{D}_{0} \stackrel{d_{0}}{\longrightarrow} \cdots \stackrel{d_{N-1}}{\longrightarrow} \mathcal{D}_{N} \rightarrow 0
$$

Lemma 3.6. Let $(\mathcal{C}, d,\langle\rangle$,$) be a Hilbert complex as in (3.41) and let k \in \mathbb{N}$, $0<k<N$. Let us denote by $\Delta$ the Laplacian associated to the Hilbert complex and by $\left(\mathcal{C}^{*}, d^{*},\langle\rangle,\right)$ the dual Hilbert complex. Assume that $0 \notin \operatorname{spec}_{\mathrm{ess}}\left(\Delta^{(i)}\right)$ for all $i \neq k$. Then

(a) range $d_{i}$ and range $d_{i}^{*}$ are closed for all $i$.

(b) If $0 \in \operatorname{spec}_{\mathrm{ess}}\left(\Delta^{(k)}\right)$, then it is an eigenvalue of infinite multiplicity.

Proof. (a) We denote by $\mathcal{D}_{i}^{*}$ (resp. $\mathcal{R}_{i}^{*}$ ) the domain (resp. the range) of $d_{i}^{*}$. We will first show by an induction that $\mathcal{R}_{i}, \mathcal{R}_{i}^{*}$ are closed for $0 \leq i<k$. Let us define the operator $A_{0}$ by

$$
A_{0}:=d_{0}: H_{0} \longrightarrow H_{1}, u_{0} \mapsto d_{0} u_{0} .
$$

The operator $A_{0}$ is a closed operator with adjoint $A_{0}^{*}=d_{0}^{*}$. Since by assumption $0 \notin \operatorname{spec}_{\text {ess }}\left(A_{0}^{*} A_{0}\right)=\operatorname{spec}_{\text {ess }}\left(\Delta^{(0)}\right)$ we deduce that $\operatorname{range}\left(A_{0}^{*} A_{0}\right)=\operatorname{range}\left(A_{0}^{*}\right)=\mathcal{R}_{0}^{*}$ is closed. Then by the closed range theorem, range $\left(A_{0}\right)=\mathcal{R}_{0}$ is also closed.

Let $0<i<k$ and let us assume that $\mathcal{R}_{j}, \mathcal{R}_{j}^{*}$ are closed for all $j<i$. We define the closed operator $A_{i}$ by

$$
A_{i}: \mathcal{D}_{i} \cap \mathcal{D}_{i-1}^{*} \subset H_{i} \longrightarrow H_{i-1} \oplus H_{i+1}, \quad u \mapsto\left(d_{i-1}^{*} u, d_{i} u\right) .
$$

By assumption $0 \notin \operatorname{spec}_{\text {ess }}\left(A_{i}^{*} A_{i}\right)=\operatorname{spec}_{\text {ess }}\left(\Delta^{(i)}\right)$. Therefore $\operatorname{range}\left(A_{i}^{*} A_{i}\right)=$ range $\left(A_{i}^{*}\right)=\mathcal{R}_{i-1} \oplus \mathcal{R}_{i}^{*}$ is closed. Using the induction hypothesis and the closed range theorem we deduce that $\mathcal{R}_{i}$ and $\mathcal{R}_{i}^{*}$ are closed.

Dually one can now consider the operator $B_{N-1}:=d_{N-1}^{*}: \mathcal{D}_{N-1}^{*} \rightarrow H_{N-1}$ and get that $\mathcal{R}_{N-1}$ and $\mathcal{R}_{N-1}^{*}$ are closed. By a downward induction one can show that $\mathcal{R}_{i}, \mathcal{R}_{i}^{*}$ are closed for $k \leq i \leq N-1$. 
(b) For any Hilbert complex the weak Hodge decomposition holds. Using in addition Part (a) of the lemma we get that

$$
H_{k}=\mathcal{H}_{k} \oplus \operatorname{range}\left(d_{k-1}\right) \oplus \operatorname{range}\left(d_{k}^{*}\right),
$$

where $\mathcal{H}_{k}:=\operatorname{ker} \Delta^{(k)}=\operatorname{ker} d_{k} \cap \operatorname{ker} d_{k-1}^{*}$. To prove the claim it is enough to show that $\left.\Delta^{(k)}\right|_{\text {range }\left(d_{k-1}\right) \oplus \operatorname{range}\left(d_{k}^{*}\right)} \geq c$ for some constant $c>0$. This can be seen as follows: Let $0 \neq \alpha \in \operatorname{range}\left(d_{k-1}\right)$. Then $\alpha=d_{k-1} \beta$ for some $\beta \in H_{k-1}$. Without loss of generality we can assume that $\beta$ is coclosed. Then by the Cauchy-Schwarz inequality

$$
\langle\Delta \alpha, \alpha\rangle=\langle\Delta d \beta, d \beta\rangle=\left\|d^{*} d \beta\right\|^{2}=\|\Delta \beta\|^{2} \geq \frac{\langle\Delta \beta, \beta\rangle^{2}}{\|\beta\|^{2}} .
$$

Since $0 \notin \operatorname{spec}_{e s s}\left(\Delta^{(k-1)}\right)$ and since $\beta$ is orthogonal to $\mathcal{H}_{k-1}$ there exists a constant $c$ such that

$$
\langle\Delta \beta, \beta\rangle \geq c\|\beta\|^{2} .
$$

From (3.45) and (3.46) we then get

$$
\langle\Delta \alpha, \alpha\rangle \geq c\langle\Delta \beta, \beta\rangle=c\|d \beta\|^{2}=c\|\alpha\|^{2} .
$$

A similar argument works for $\alpha \in \operatorname{range}\left(d_{k}^{*}\right)$.

Proof of Theorem 3.1 b). Note that by Theorem 2.4 in $[3$ the Fredholm property of the complex $\left(\mathcal{D}_{t}, d_{t},\langle\rangle,\right)$ is equivalent to $0 \notin \operatorname{spec}_{\text {ess }}\left(\boldsymbol{\Delta}_{t}\right)$. Once the Fredholm property is proved, the isomorphism in (3.8) follows from standard arguments on the Hodge theory of Hilbert complexes (see [3], Corollary 2.5).

Let us show first that $0 \notin \operatorname{spec}_{\text {ess }}\left(\boldsymbol{\Delta}_{t}^{(i)}\right)$ for $i \neq \nu$ : By the rescaling argument in Lemma 3.3 it is enough to prove $0 \notin \operatorname{spec}_{\text {ess }}\left(\boldsymbol{\Delta}_{1}^{(i)}\right)$. Since $U^{-1} \boldsymbol{\Delta}_{1}^{(i)} U=\mathbb{T}_{1}^{(i)}$, this is equivalent to proving that $0 \notin \operatorname{spec}_{\mathrm{ess}}\left(\mathbb{T}_{1}^{(i)}\right)$. But for $i \neq \nu$ we have $\mathbb{T}_{1}^{(i)}=\mathbb{T}_{1}^{(i), \mathcal{F}}$ (this can be seen as in Lemma 2.5). The claim follows using Lemma 3.5 (c).

Now we consider the case $i=\nu$ : Let us assume that $0 \in \operatorname{spec}_{\mathrm{ess}}\left(\boldsymbol{\Delta}_{t}^{(\nu)}\right)$. By the first part of the proof we know that the assumptions of Lemma 3.6 hold for the Hilbert complex $\left(\mathcal{D}_{t}, d_{t},\langle\rangle,\right)$ (and $\left.k=\nu\right)$. Thus we conclude that 0 is an eigenvalue of $\boldsymbol{\Delta}_{t}^{(\nu)}$ of infinite multiplicity. Let $\left\{\phi_{n}\right\}_{n \in \mathbb{N}}$ be a sequence of orthogonal eigenforms in ker $\boldsymbol{\Delta}_{t}^{(\nu)}$. Denote by $P_{t}^{\nu}$ the Dirichlet realisation of the Witten Laplacian on the truncated cone cone $_{1}(L)$. (More precisely for $P_{t}^{\nu}$, we impose Dirichlet boundary conditions at $r=1$ and the conditions in (3.11) locally near the singularity.) This is a non-negative operator with finitely many eigenvalues in each finite interval. Let $\chi:$ cone $(L) \rightarrow[0,1]$ be a cut-off function with supp $\chi \subset$ cone $_{1 / 2}(L)$. Using Agmon type estimates similar to those in [14] one can prove that for $t$ large enough we get

$$
\left\langle P_{t}^{\nu}\left(\chi \phi_{n}\right), \chi \phi_{n}\right\rangle=O\left(e^{-c t}\right)\left\|\chi \phi_{n}\right\|^{2} .
$$

Using the min-max principle this gives a contradiction to the fact that $P_{t}^{\nu}$ has only finitely many eigenvalues in the interval, say e.g. [0,1]. Therefore we have shown that $0 \notin \operatorname{spec}_{\text {ess }}\left(\boldsymbol{\Delta}_{t}^{(\nu)}\right)$ for sufficiently large $t$. By rescaling we get $0 \notin \operatorname{spec}_{\text {ess }}\left(\boldsymbol{\Delta}_{t}^{(\nu)}\right)$ for all $t>0$. 
3.6. Proof of Theorem $\mathbf{3 . 1}$ (c). Note that $\boldsymbol{\Delta}_{1}$ is a non-negative operator. The proof of part (b) showed that $0 \notin \operatorname{spec}_{e s s}\left(\boldsymbol{\Delta}_{1}\right)$. Thus there exists a constant $c>0$ such that $\operatorname{spec}\left(\boldsymbol{\Delta}_{1}\right) \subset\{0\} \cup[c, \infty)$. By the rescaling argument in Lemma 3.3 we deduce that $\operatorname{spec}\left(\boldsymbol{\Delta}_{t}\right) \subset\{0\} \cup\left[c t^{2}, \infty\right)$. The claim on the decay of the eigenfunctions follows from Agmon type estimates similar to those in [14] (see also [19] for Agmon type estimates for a singular curve).

3.7. Proof of Theorem 3.1 (d). To prove Theorem 3.1 (d) we adapt the cone construction proposed in [7], Section 5 (resp. [11, Section 3) in the context of the Witten deformation on manifolds with boundary (resp. of the Witten deformation for polynomial differential forms on non-compact manifolds). The proof follows closely the lines of proof in [7, [11]; one has to take care that the arguments go through at the cone point. We give the arguments of the proof in some detail for the convenience of the reader.

Denote by $\left(\mathcal{E}^{*}, d,\langle\rangle,\right)$ the complex defined by

$$
\mathcal{E}^{i}:=\left\{\omega \in \Omega^{*}(\operatorname{cone}(L) \backslash\{p\}) \mid \omega, d \omega \in \mathrm{L}^{2} \text { loc. at } p\right\} .
$$

Recall that dim cone $(L)=2 \nu$. It is not difficult to see (compare the remark below) that the complex $\left(\mathcal{E}^{*}, d,\langle\rangle,\right)$ computes the $\mathrm{L}^{2}$-cohomology of the punctured cone,

$$
H^{*}\left(\mathcal{E}^{*}, d,\langle,\rangle\right) \simeq H_{(2)}^{*}(\operatorname{cone}(L))= \begin{cases}H_{(2)}^{i}(L)=H^{i}(L), & i \leq \nu-1, \\ 0, & \text { else }\end{cases}
$$

For cones the integration morphism gives a duality between $\mathrm{L}^{2}$-cohomology and intersection homology with middle perversity. Thus

$$
H^{*}\left(\mathcal{E}^{*}, d,\langle,\rangle\right) \simeq I H^{*}(\operatorname{cone}(L)) .
$$

For $c>0$ let $j_{c}: U_{c}^{-} \subset \operatorname{cone}(L) \backslash\{p\}$ be the open subset of cone $(L)$ defined by

$$
U_{c}^{-}:=\{(r, \varphi) \in \operatorname{cone}(L) \mid f(r, \varphi)<-c\} .
$$

Note that for $c^{\prime}>c$ we have $U_{c^{\prime}}^{-} \subset U_{c}^{-}$. Moreover

$$
\operatorname{int}\left(l^{-}\right) \subset U_{c^{\prime}}^{-} \subset U_{c}^{-}
$$

are deformation retracts. We recall that $l^{-}$is the lower half-link defined in Section 3.2 (choosing $\delta>c^{\prime}>c$ in the construction there) and we denote by $\operatorname{int}\left(l^{-}\right):=$ $l^{-} \backslash \partial l^{-}$. We denote by $\left(\Omega^{*}\left(U_{c}^{-}\right), d\right)$ the de Rham complex of smooth forms on $U_{c}^{-}$. Using the de Rham isomorphism between singular cohomology and de Rham cohomology as well as the homotopy invariance of singular cohomology we get

$$
H^{*}\left(\Omega^{*}\left(U_{c}^{-}\right), d\right)=H_{d R}^{*}\left(U_{c}^{-}\right) \simeq H_{\text {sing }}^{*}\left(U_{c}^{-}\right) \simeq H_{\text {sing }}^{*}\left(l^{-}\right) .
$$

Since $l^{-}$is smooth we have moreover that $H_{\text {sing }}^{*}\left(l^{-}\right) \simeq I H^{*}\left(l^{-}\right)$and therefore

$$
H^{*}\left(\Omega^{*}\left(U_{c}^{-}\right), d\right) \simeq I H^{*}\left(l^{-}\right) .
$$

We denote by $j_{c}^{*}$ the restriction map

$$
j_{c}^{*}: \mathcal{E}^{i} \rightarrow \Omega^{i}\left(U_{c}^{-}\right), \omega \mapsto \omega_{\mid U_{c}^{-}} .
$$

The cone complex $\operatorname{Cone}\left(j_{c}^{*}\right)$ is defined as follows (see e.g. [10]):

$$
\operatorname{Cone}^{k}\left(j_{c}^{*}\right)=\mathcal{E}^{k} \oplus \Omega^{k-1}\left(U_{c}^{-}\right), d_{\text {Cone }}\left(\eta, \eta^{\prime}\right)=\left(d \eta,-d \eta^{\prime}+j_{c}^{*} \eta\right) .
$$


By construction of the cone complex and in view of (3.51) and (3.55) we get

$$
H^{*}\left(\operatorname{Cone}\left(j_{c}^{*}\right)\right) \simeq I H^{*}\left(\operatorname{cone}(L), l^{-}\right) .
$$

Note that because of (3.53) the isomorphism (3.58) holds for all $c>0$.

Remark 3.7. Some remarks may be in order here:

(1) While all forms in the complex $\left(\mathcal{D}_{t}, d_{t},\langle\rangle,\right)$ are $\mathrm{L}^{2}$-integrable on the infinite cone, for the complex $(\mathcal{E}, d,\langle\rangle$,$) we allow forms which are not necessarily$ $\mathrm{L}^{2}$-integrable at $\infty$.

(2) In 9] the complex of $\mathrm{L}^{2}$-forms on the truncated cone cone ${ }_{1}(L)$ is used to compute the $\mathrm{L}^{2}$-cohomology of the punctured cone. Here we use the complex $(\mathcal{E}, d,\langle\rangle$,$) on the infinite cone instead. However to prove (3.50)$ one can proceed as in 9 and construct homotopy operators which contract to the tip point (resp. to the base $L$ of the cone) if the form degree is $i \geq \nu$ (resp. $i<\nu$ ).

Let $\left(\mathcal{D}_{t}^{\infty}, d_{t},\langle\rangle,\right)$ be the subcomplex of $\left(\mathcal{D}_{t}, d_{t},\langle\rangle,\right)$ of smooth forms, i.e. $\mathcal{D}_{t}^{\infty, i}:=\mathcal{D}_{t}^{i} \cap \Omega^{i}(\operatorname{cone}(L) \backslash\{p\})$. By the usual regularisation argument

$$
H^{*}\left(\mathcal{D}_{t}^{\infty}, d_{t},\langle,\rangle\right) \simeq H^{*}\left(\mathcal{D}_{t}, d_{t},\langle,\rangle\right) .
$$

Let us recall that a form $\omega \in \mathcal{D}_{t}^{\infty}$ can be decomposed as

$$
\omega=\omega_{\perp}+d r \wedge \omega_{\|},
$$

where $\omega_{\perp}$ and $\omega_{\|}$are forms on the link depending smoothly on $r$.

Definition/Lemma 3.8. We can define the following chain map:

$$
\begin{aligned}
& \Phi:\left(\mathcal{D}_{t}^{\infty}, d_{t},\langle,\rangle\right) \quad \longrightarrow\left(\operatorname{Cone}\left(j_{c}^{*}\right), d_{\text {Cone }}\right) \\
& \omega=\omega_{\perp}+d r \wedge \omega_{\|} \quad \mapsto \quad\left(e^{t f} \omega, \omega^{\prime}\right),
\end{aligned}
$$

where

$$
\omega^{\prime}(r, \varphi)=-\int_{r}^{\infty} e^{t f(\tau, \varphi)} \omega_{\|}(\tau, \varphi) d \tau \text { for }(r, \varphi) \in U_{c}^{-}
$$

Proof. Note first that since $f<-c$ on $U_{c}^{-}$and $\omega \in \mathcal{D}_{t}^{\infty}$ the integral in (3.62) converges. Moreover to check that $\Phi$ is a chain map one has to verify that, on $U_{c}^{-}$,

$$
-\int_{r}^{\infty} e^{t f}\left(d_{t} \omega\right)_{\|} d \tau=d\left(\int_{r}^{\infty} e^{t f} \omega_{\|} d \tau\right)+e^{t f} \omega
$$

As in Section 3.3, let us denote by $\tilde{d}$ the operator on the link $L$. The equation (3.63) follows from the following computation:

$$
\begin{aligned}
-\int_{r}^{\infty} e^{t f}\left(d_{t} \omega\right)_{\|} d \tau & =-\int_{r}^{\infty} e^{t f}(d \omega+t d f \wedge \omega)_{\|} d \tau \\
& =-\int_{r}^{\infty} e^{t f}\left(\frac{\partial \omega_{\perp}}{\partial \tau}-\tilde{d} \omega_{\|}-t \tau d h \wedge \omega_{\|}+t h \omega_{\perp}\right) d \tau \\
& =-\int_{r}^{\infty} \frac{\partial}{\partial \tau}\left(e^{t f} \omega_{\perp}\right) d \tau+\int_{r}^{\infty} \tilde{d}\left(e^{t f} \omega_{\|}\right) d \tau \\
& =e^{t f} \omega_{\perp}+d r \wedge e^{t f} \omega_{\|}+d\left(\int_{r}^{\infty} e^{t f} \omega_{\|} d \tau\right)
\end{aligned}
$$


In view of (3.58) and (3.59), in order to prove Theorem 3.1)(d) it is enough to prove the following proposition:

Proposition 3.9. The chain map $\Phi$ defined in (3.61) is a quasi-isomorphism.

Proof. To prove the proposition we just have to check that the arguments in [7], [11] adapt to our singular situation. We denote by $\Phi^{*}: H^{*}\left(\mathcal{D}_{t}^{\infty}, d_{t},\langle\rangle,\right) \rightarrow$ $H^{*}\left(\right.$ Cone $\left.\left(j_{c}^{*}\right), d_{\text {Cone }}\right)$ the induced map on the cohomology level. First we prove the injectivity of $\Phi^{*}$ :

Let $[\omega] \in H^{*}\left(\mathcal{D}_{t}^{\infty}, d_{t},\langle\rangle,\right)$ with $\Phi^{*}[\omega]=0$. In view of the Hodge theory for the complex $\left(\mathcal{D}_{t}, d_{t},\langle\rangle,\right)$ (part (b) of Theorem 3.1) and elliptic regularity we can choose a representative $\omega \in \operatorname{ker} \boldsymbol{\Delta}_{t}$ for the class $[\omega]$. The fact that $\Phi^{*}[\omega]=0$ means that there exists $\left(\alpha, \alpha^{\prime}\right) \in \mathcal{E}^{i-1} \oplus \Omega^{i-2}\left(U_{c}^{-}\right)$such that

$$
\begin{aligned}
& d \alpha=e^{t f} \omega, \\
& -d \alpha^{\prime}+j_{c}^{*} \alpha=\omega^{\prime} .
\end{aligned}
$$

To show injectivity of $\Phi^{*}$ we have to find $\zeta \in \mathcal{D}_{t}^{\infty, i-1}=\operatorname{dom}\left(d_{t, i-1}\right)$ with $d_{t} \zeta=\omega$. This will be done by modifying the form $\alpha$ as in [7. The main point is to notice that the proof also works in our situation since all the modifications are done outside a small neighbourhood of the cone tip.

Step 1. Let $c<c^{\prime}$. Let $\chi_{1}: \operatorname{cone}(L) \rightarrow[0,1]$ be a cutoff function such that $\chi_{1 \mid U_{c^{\prime}}^{-}}=1$ and $\chi_{1 \mid \operatorname{cone}(L) \backslash U_{c}^{-}}=0$.

Set

$$
\beta:=\alpha-d\left(\chi_{1} \alpha^{\prime}\right)
$$

Then we get

$$
\begin{aligned}
& d \beta=e^{t f} \omega \text { directly from the definition of } \beta \text { and (3.65a), } \\
& \beta_{\mid U_{c^{\prime}}^{-}}=\omega^{\prime} \text { from the definition of } \beta \text { and (3.65b). }
\end{aligned}
$$

Step 2. Let $\chi_{2}: \operatorname{cone}(L) \rightarrow[0,1]$ be a cutoff function such that $\chi_{2}=1$ for $r \geq 2$ and $\chi_{2}=0$ for $r \leq 1$. Set

$$
\gamma:=\beta-d\left(\chi_{2} \int_{2}^{r} \beta_{\|} d \tau\right)
$$

Then we get by using (3.67a) and (3.68) that

$$
\begin{aligned}
d \gamma & =e^{t f} \omega, \\
\gamma_{||} & =0 \text { on } r \geq 2 .
\end{aligned}
$$

From (3.69a) and (3.69b) we get in particular that $d \gamma_{\perp}=e^{t f} \omega$ and therefore

$$
\frac{\partial}{\partial r} \gamma_{\perp}=e^{t f} \omega_{\|} \text {for } r \geq 2
$$

Step 3. For $\epsilon \in \mathbb{R}$ set $S_{\epsilon}:=\left\{\varphi \in L \mid h(\varphi)<\frac{\epsilon}{t}\right\}$. From Proposition 3.1(c) we know that all forms in ker $\boldsymbol{\Delta}_{t}$ have exponential decay; thus there exist constants $a>0$, $C>0$ such that

$$
\omega \leq C e^{-a r}
$$

outside a small neighbourhood of the singularity. Hence

$$
e^{t f} \omega \leq C e^{-a r+\epsilon r} \text { if }(r, \varphi) \in S_{\epsilon} \times[2, \infty) .
$$


Therefore the integral $\int_{r}^{\infty} e^{t f} \omega_{\|} d \tau$ is well defined and the differential equation (3.70) on $S_{a} \times[2, \infty)$ is solved by

$$
\gamma_{\perp}=\xi-\int_{r}^{\infty} e^{t f} \omega_{\|} d \tau
$$

where $\xi$ is a differential form on $S_{a}$ (which does not depend on the radial coordinate $r)$.

From (3.69a) and (3.73) we get that

$$
e^{t f} \omega_{\perp}=\tilde{d} \gamma_{\perp}=\tilde{d} \xi-\int_{r}^{\infty} \tilde{d}\left(e^{t f} \omega_{\|}\right) d \tau, \text { on } S_{a} \times[2, \infty) .
$$

Now since $d_{t} \omega=0$ we have

$$
0=\left(d_{t} \omega\right)_{\|}=e^{-t f}\left(-\tilde{d}\left(e^{t f} \omega_{\|}\right)+\frac{\partial}{\partial r}\left(e^{t f} \omega_{\perp}\right)\right)
$$

and thus

$$
-\tilde{d}\left(e^{t f} \omega_{\|}\right)+\frac{\partial}{\partial r}\left(e^{t f} \omega_{\perp}\right)=0 .
$$

Inserting (3.76) into (3.74) we get that $\left(\right.$ on $\left.S_{a} \times[2, \infty)\right)$

$$
e^{t f} \omega_{\perp}=\tilde{d} \xi+e^{t f} \omega_{\perp}
$$

and thus $\tilde{d} \xi=0$; i.e. $\xi$ is a closed form on $S_{a}$.

An explicit computation using (3.67b), (3.68) and (3.73) shows that

$$
\xi=-d\left(\left(\int_{2}^{\infty} e^{t f} \beta_{\|}\right) \text {on } U_{c^{\prime}}^{-}\right.
$$

Note that for $c^{\prime}$ large enough, $U_{c^{\prime}}^{-} \subset\{r \geq 2\}$ and $\xi$ does not depend on the radial coordinate $r$. Moreover for each $\varphi \in S_{0}$ there exists $r_{\varphi}$ such that $\left(r_{\varphi}, \varphi\right) \in U_{c^{\prime}}^{-}$. We conclude that $\xi_{\mid S_{0}}$ is exact. Thus $[\xi]=0 \in H^{*}\left(S_{0}\right)$. Since by definition of an admissible Morse function (see also (3.27)) 0 is a regular value of $h$ we can find $\epsilon \in(0, a / 2)$ such that $H^{*}\left(S_{0}\right) \simeq H^{*}\left(S_{2 \epsilon}\right)$. Thus $[\xi]=0 \in H^{*}\left(S_{2 \epsilon}\right)$; i.e. there exists a form $\mu \in \Omega^{*}\left(S_{2 \epsilon}\right)$ with $d \mu=\xi$. Let $\chi_{3}: L \rightarrow[0,1]$ be a cutoff function with $\chi_{3 \mid S_{4 / 3 \epsilon}} \equiv 1$ and $\chi_{3 \mid L \backslash S_{5 / 3 \epsilon}} \equiv 0$. Set

$$
\theta:=\gamma-d\left(\chi_{3} \chi_{2} \mu\right)
$$

We have

$$
\begin{aligned}
& d \theta=d \gamma=e^{t f} \omega \\
& \theta_{\mid S_{\epsilon} \times[2, \infty)}=\gamma_{\perp}-d \mu=-\int_{r}^{\infty} e^{t f} \omega_{\|} d \tau \text { by using (3.73), } \\
& \left.\theta_{\|}=0 \text { on } r>2 \text { by the definition of } \theta \text { and } 3.69 \mathrm{~b}\right) .
\end{aligned}
$$

Set $\zeta:=e^{-t f} \theta$. Then $d_{t} \zeta=\omega$. The proof of the injectivity of $\Phi^{*}$ is completed if we can show that $\zeta \in \mathcal{D}_{t}^{\infty}=\operatorname{dom}\left(d_{t}\right)$. Since $d_{t} \zeta=\omega \in \mathrm{L}^{2}$ we need to show only that $\zeta \in \mathrm{L}^{2}\left(\Lambda^{*}\left(T^{*}(\operatorname{cone}(L))\right)\right.$. Note first that since the cutoff functions $\chi_{1}, \chi_{2}$ used to construct $\theta$ from $\alpha$ have support outside the cone point and $\alpha \in \mathrm{L}^{2}$ locally near the cone point we have $\zeta_{\mid \operatorname{cone}_{2}(L)} \in \mathrm{L}^{2}$. One uses (3.72) and (3.79b) to show 
that $\zeta_{\mid S_{\epsilon} \times[2, \infty)}$ has exponential decay for $r \rightarrow \infty$ and thus $\zeta_{\mid S_{\epsilon} \times[2, \infty)} \in \mathrm{L}^{2}$. On $\left(L \backslash S_{\epsilon}\right) \times[2, \infty)$ we use (3.79a) and (3.79c) and thus

$$
\frac{\partial}{\partial r} \theta_{\perp}=e^{t f} \omega_{\|}
$$

By integrating (3.80) one gets

$$
\theta_{\perp}=\theta_{\perp}(\varphi, 2)+\int_{2}^{r} e^{t f} \omega_{\|} d \tau
$$

Again using (3.71) and (3.81) one gets that $\zeta_{\mid\left(L \backslash S_{\epsilon}\right) \times[2, \infty)}$ is exponentially decaying and thus $\zeta_{\mid\left(L \backslash S_{\epsilon}\right) \times[2, \infty)} \in \mathrm{L}^{2}$. This finishes the proof of the injectivity of $\Phi^{*}$.

We now show the surjectivity of $\Phi^{*}$ : We can choose $c>0$ large enough such that $U_{c}^{-} \subset S_{\epsilon} \times[3, \infty)$. Moreover $U_{c}^{-}$is a deformation retract of $S_{\epsilon} \times[3, \infty)$. As in [7] one can show that a class $\left[\left(\alpha, \alpha_{1}\right)\right] \in H^{i}\left(\operatorname{Cone}\left(j_{c}^{*}\right)\right)$ can be represented by a pair $(\gamma, 0) \in \mathcal{E}^{i} \oplus \Omega^{i-1}\left(U_{c}^{-}\right)$such that $d \gamma=0,\left.\gamma\right|_{S_{\epsilon} \times[3, \infty)}=0$ and $\gamma$ is bounded. We then have $e^{-t f} \gamma \in \mathrm{L}^{2}$ : Since $\gamma \in \mathcal{E}^{i}$, in particular $\left.e^{-t f} \gamma\right|_{\text {cone }_{3}(L)} \in \mathrm{L}^{2}$. Moreover $\left.\gamma\right|_{S_{\epsilon} \times[3, \infty)}=0$ and on $\left(L \backslash S_{\epsilon}\right) \times[3, \infty)$ we have $e^{-t f}<e^{-t \epsilon r}$. Since $\gamma$ is bounded we deduce that $\left.e^{-t f} \gamma\right|_{\left(L \backslash S_{\epsilon}\right) \times[3, \infty)} \in \mathrm{L}^{2}$. We conclude that $e^{-t f} \gamma \in \mathcal{D}_{t}^{\infty, i}$ and moreover $\Phi^{*}\left(\left[e^{-t f} \gamma\right]\right)=[(\gamma, 0)]=\left[\left(\alpha, \alpha_{1}\right)\right]$.

Example 3.10. Let us illustrate the result in Theorem 3.1 for the case $f= \pm r$. In this case we have separation of variables for the model Witten Laplacian $\boldsymbol{\Delta}_{t}$ and the lower half-link is easy to determine. Therefore we can compute both sides of the isomorphism

$$
\operatorname{ker}\left(\boldsymbol{\Delta}_{t}^{(i)}\right) \simeq I H^{i}\left(\operatorname{cone}(L), l^{-}\right)
$$

explicitly. In the following we denote by $\mathcal{H}^{i}(L)$ the harmonic $i$-forms on the link $L$.

(a) Let $f=r$. We get, with the notation as in Section 3.3 (for the first order operator acting on $i$-forms)

$$
U^{-1} \mathbf{D}_{t} U= \pm \partial_{r}+r^{-1}\left(\begin{array}{cc}
c_{i-1} & \widetilde{\delta} \\
\widetilde{d} & c_{i}
\end{array}\right)+t\left(\begin{array}{cc}
(-1)^{i-1} & 0 \\
0 & (-1)^{i}
\end{array}\right)
$$

and an explicit computation shows that

$$
\operatorname{ker} \Delta_{t}^{(i)}=V_{+}^{i}
$$

where

$$
V_{+}^{i}:= \begin{cases}\operatorname{span}\left\{e^{-t r} \eta \mid \eta \in \mathcal{H}^{i}(L)\right\} & \text { if } i<\nu \\ 0 & \text { if } i \geq \nu .\end{cases}
$$

Note that for $i<\nu$ the forms in $V_{+}^{i}$ are locally $\mathrm{L}^{2}$ near the cone point, and the factor $e^{-t r}$ ensures that they are $L^{2}$ at $\infty$. Recall that dim cone $(L)=$ $2 \nu$. On the other hand $l^{-}=\emptyset$ and from the local calculation for intersection homology with middle perversity (see [12]) we know that

$$
I H^{i}(\operatorname{cone}(L), \emptyset)=I H^{i}(\operatorname{cone}(L))= \begin{cases}H^{i}(L) & \text { if } i<\nu \\ 0 & \text { if } i \geq \nu\end{cases}
$$

Since by Hodge theory for the smooth manifold $L$ we have $\mathcal{H}^{i}(L) \simeq$ $H^{i}(L)$, the isomorphism (3.82) follows from (3.84) and (3.86). 
(b) $f=-r$. Again an explicit computation shows that

$$
\operatorname{ker}\left(\boldsymbol{\Delta}_{t}^{(i)}\right)=V_{-}^{i}
$$

where

$$
V_{-}^{i}:= \begin{cases}0 & \text { if } i \leq \nu \\ \operatorname{span}\left\{e^{-t r} r^{-n+2(i-1)} d r \wedge \eta \mid \eta \in \mathcal{H}^{i-1}(L)\right\} & \text { if } i>\nu .\end{cases}
$$

Note again that the form $e^{-t r} r^{-n+2(i-1)} d r \wedge \eta, h \in H^{i-1}(L)$, is $\mathrm{L}^{2}$-integrable if and only if $i>\nu$.

On the other hand we have $l^{-}=L$ and by the local calculation for the intersection homology with middle perversity,

$$
I H^{i}(\operatorname{cone}(L), L)= \begin{cases}0 & \text { if } i \leq \nu, \\ H^{i-1}(L) & \text { if } i>\nu .\end{cases}
$$

Again (3.87) and (3.89) show the isomorphism (3.82) explicitly for this case.

Note that comparing (a) and (b) one recognises the usual Poincaré duality in Morse theory, namely that the Hodge star operator $*$ yields an isomorphism

$$
*: V_{-}^{i} \simeq V_{+}^{2 \nu-i}
$$

Example 3.11. In 20] the case of a stratified Morse function in the sense of Goresky and MacPherson on a singular complex curve has been treated. In this case the local model for a (unibranched) singular point $p$ of multiplicity $m$ is given by (cone $\left(S_{m}^{1}\right), d r^{2}+r^{2} d \varphi^{2}$ ), where $S_{m}^{1}$ denotes the circle of length $2 \pi m$. A stratified Morse function (in the coordinates $(r, \varphi)$ ) is just $f=r \cos \varphi$. The local model for the Witten Laplacian is then " $\boldsymbol{\Delta}_{t}=\boldsymbol{\Delta}+t^{2}$ " (but $\operatorname{dom}\left(\boldsymbol{\Delta}_{t}\right) \neq \operatorname{dom}(\boldsymbol{\Delta})$ !) and an explicit computation shows that

$$
\operatorname{dim} \operatorname{ker} \boldsymbol{\Delta}_{t}=\operatorname{dim} \operatorname{ker} \boldsymbol{\Delta}_{t}^{(1)}=m-1 .
$$

The elements in ker $\boldsymbol{\Delta}_{t}$ involve the modified Bessel functions $K_{\nu}$, where $\nu^{2} \in(0,1)$ is an eigenvalue in $\operatorname{spec}\left(\Delta_{S_{m}^{1}}\right)=\left\{0, \frac{1}{m^{2}}, \frac{4}{m^{2}}, \ldots\right\}$. On the other hand, here the lower half-link $l^{-}$is homotopic to $m$ points and therefore

$$
\operatorname{dim} I H^{i}\left(\operatorname{cone}\left(S_{m}^{1}\right), l^{-}\right)= \begin{cases}0 & \text { if } i=0,2 \\ m-1 & \text { if } i=1\end{cases}
$$

\section{Proof of the spectral gap theorem and Morse inequalities}

Proof of Theorem 1.1(1). The proof of the spectral gap theorem consists of two steps. The first step, namely the study of a model operator for the Witten Laplacian in the neighbourhood of a singular point $p \in \Sigma$ of $X$, has been done in Section 3. In the second step of the proof it is now enough to follow the strategy of proof in the smooth case. Here we follow the proof in [1, Section 9 and just give a very rough outline. (In [20] the proof has been detailed for the case of a complex curve with cone-like singularities.) Recall from the smooth theory that the model Witten Laplacian $\boldsymbol{\Delta}_{t, p}$ in the neighbourhood of a critical point $p \in X \backslash \Sigma$ of $f$ of index $i$ has discrete spectrum $\operatorname{spec}\left(\boldsymbol{\Delta}_{t, p}\right)=2 \mathbb{N} t$ and $\operatorname{dim} \operatorname{ker}\left(\boldsymbol{\Delta}_{t, p}\right)=\operatorname{dim} \operatorname{ker}\left(\boldsymbol{\Delta}_{t, p}^{(i)}\right)=1$. We denote by $\omega_{p, 1}^{i}(t)$ the generator of $\operatorname{ker}\left(\boldsymbol{\Delta}_{t, p}\right)$. For a singular point $p \in \Sigma$ we denote by $\left\{\omega_{p, j}^{i}(t) \mid j=1, \ldots, m_{p}^{i}\right\}$ a basis of $\operatorname{ker}\left(\boldsymbol{\Delta}_{t, p}^{(i)}\right), i=0, \ldots, 2 \nu$. Let $\nu_{\epsilon}: \mathbb{R}^{+} \rightarrow \mathbb{R}$ 
be a cutoff function with $\nu_{\epsilon}=1$ in $[0, \epsilon / 4]$ and $\operatorname{supp} \nu_{\epsilon} \subset[0, \epsilon / 2](\epsilon$ as in Section 3). The forms $\Phi_{p, j}^{i}(t):=\nu_{\epsilon}(|x|) \omega_{p, j}^{i}(t)$ can be identified with $\mathrm{L}^{2}$-forms on $X$. For $i=0, \ldots, 2 \nu$ we denote by

$E^{i}(t):=\operatorname{span}\left\{\left\{\Phi_{p, 1}^{i}(t) \mid p \in \operatorname{Crit}_{i}(f) \backslash \Sigma\right\} \cup\left\{\Phi_{p, j}^{i}(t) \mid p \in \Sigma, j \in I_{p}^{i}:=\left\{1, \ldots, m_{p}^{i}\right\}\right\}\right\}$.

We get an orthogonal splitting $\mathrm{L}^{2}\left(\Lambda^{*}\left(T^{*}(X \backslash \Sigma)\right)\right)=E(t) \oplus E(t)^{\perp}$. The closed operator $D_{t}:=d_{t}+\delta_{t}$ with $\operatorname{dom}\left(D_{t}\right)=\operatorname{dom}\left(d_{t}\right) \cap \operatorname{dom}\left(\delta_{t}\right) \subset \mathrm{L}^{2}\left(\Lambda^{*}\left(T^{*}(X \backslash \Sigma)\right)\right)$ can be written in matrix form

$$
D_{t}=\left(\begin{array}{cc}
D_{t, 1} & D_{t, 2} \\
D_{t, 3} & D_{t, 4}
\end{array}\right) \text { according to the splitting } E(t) \oplus E(t)^{\perp} .
$$

Note that $\operatorname{dom}\left(D_{t}\right)$ equipped with the norm $\|\omega\|_{1}:=\sqrt{\|(d+\delta) \omega\|^{2}+\|\omega\|^{2}}$ is complete. We show the following estimates on $D_{t}$ as $t \rightarrow \infty$ :

Proposition 4.1. There exist constants $c, C>0$ and $t_{0}>0$ such that for all $t>t_{0}$ we have:

(1) For all $\omega \in E(t)$ we have $\left\|D_{t} \omega\right\|=O\left(e^{-c t}\right)\|\omega\|$. In particular $\left\|D_{t, 1} \omega\right\|=$ $O\left(e^{-c t}\right)\|\omega\|,\left\|D_{t, 3} \omega\right\|=O\left(e^{-c t}\right)\|\omega\|$.

(2) For all $\omega \in E(t)^{\perp} \cap \operatorname{dom}\left(D_{t}\right)$ we get $\left\|D_{t, 2} \omega\right\| \leq O\left(e^{-c t}\right)\|\omega\|,\left\|D_{t, 4} \omega\right\| \geq$ $C\left(\|\omega\|_{1}+\sqrt{t}\|\omega\|\right)$.

The proof of Proposition 4.1 is similar to the corresponding statements in the smooth case (see [1, Section 9). To prove the estimates for forms $\omega$ with support in a neighbourhood of a singular point of $X$ the local spectral gap theorem and the decay of eigenforms of the model operator (see Theorem 3.1 (c)) are crucial. As in [1], Section 9 (c) and (e), Proposition 4.1 allows us to give estimates for the resolvent of $D_{t}-\lambda: \operatorname{dom}\left(D_{t}\right) \rightarrow \mathrm{L}^{2}\left(\Lambda^{*}\left(T^{*}(X \backslash \Sigma)\right)\right)$, where $\lambda \in \mathbb{C},|\lambda| \in\left[e^{-c t / 2}, \frac{C \sqrt{t}}{2}\right]$, with constants $c, C$ as in Proposition 4.1. We deduce the invertibility of the operator $D_{t}-\lambda$, and since $\Delta_{t}-\lambda^{2}=\left(D_{t}-\lambda\right)\left(D_{t}+\lambda\right)$ we thus get Part (1) of Theorem 1.1 .

Proof of Part (2) of Theorem 1.1 and Corollary 1.2. For $i=0, \ldots, 2 \nu$ we define the $\mathbb{R}$-vector space $C^{i}$ by

$$
C^{i}:=\bigoplus_{p \in \operatorname{Crit}_{i}(f) \backslash \Sigma} \mathbb{R} \cdot e_{p, 1}^{i} \oplus \bigoplus_{p \in \Sigma, j \in I_{p}^{i}} \mathbb{R} \cdot e_{p, j}^{i}
$$

Note that by construction $\operatorname{dim} C^{i}=c_{i}(f)$ with $c_{i}(f)$ as in (1.4). We define a linear map

$$
J_{i}(t): C^{i} \longrightarrow \mathcal{C}_{t}^{i}, J_{i}(t)\left(e_{p, j}^{i}\right)=\Phi_{p, j}^{i}(t) .
$$

We denote by $\left(\mathcal{S}_{t}, d_{t},\langle\rangle,\right)$ the subcomplex of $\left(\mathcal{C}_{t}, d_{t},\langle\rangle,\right)$ generated by the eigenforms of $\Delta_{t}$ to eigenvalues lying in $[0,1]$. We denote moreover by $P(t,[0,1])$ the orthogonal projection operator from $\mathcal{C}_{t}$ on $\mathcal{S}_{t}$ with respect to $\langle$,$\rangle .$

As in [19], Section 4, one can now show that the linear map $P_{i}(t,[0,1]) \circ J_{i}(t)$ : $C^{i} \longrightarrow \mathcal{S}_{t}^{i}$ is a bijective map from $C^{i}$ onto $\mathcal{S}_{t}^{i}$ and thus the complex $\left(\mathcal{S}_{t}, d_{t},\langle\rangle,\right)$ is a finite dimensional subcomplex of $\left(\mathcal{C}_{t}, d_{t},\langle\rangle,\right)$ with $\operatorname{dim} \mathcal{S}_{t}^{i}=c_{i}(f)$. By Proposition 2.6 moreover $H_{(2)}^{*}(X) \simeq \operatorname{ker}\left(\Delta_{t}\right) \simeq H^{*}\left(\mathcal{S}_{t}, d_{t},\langle\rangle,\right)$. The Morse inequalities in Corollary 1.2 now follow by a standard algebraic argument. 


\section{ACKNOWLEDGEMENTS}

The author thanks Jean-Michel Bismut for suggesting work on the subject and is grateful to Jochen Brüning and Jean-Paul Brasselet for many helpful conversations, as well as to Olaf Post. A part of this work was done while the author was supported by SFB 647 .

\section{REFERENCES}

1. J. M. Bismut and G. Lebeau, Complex immersions and Quillen metrics, Publ. Math. Inst. Hautes Etud. Sci. 74 (1991), 1-197. MR1188532 (94a:58205)

2. J. M. Bismut and W. Zhang, An extension of a theorem by Cheeger and Müller. With an appendix by François Laudenbach, Astérisque 205 (1992) Société Mathématique de France.

3. J. Brüning and M. Lesch, Hilbert complexes, J. Funct. Anal. 108 (1) (1992), 88-132. MR 1174159 (93k:58208)

4. J. Brüning and M. Lesch, Kähler-Hodge theory for conformal complex cones, Geom. Funct. Anal. 3 (5) (1993), 439-473. MR1233862 (94i:58189)

5. J. Brüning and M. Lesch, On the spectral geometry of algebraic curves, J. Reine Angew. Math. 474 (1996), 25-66. MR1390691 (97d:58193)

6. J. Brüning and R. Seeley, An index theorem for first order regular singular operators, Amer. J. Math. 110 (4) (1988), 659-714. MR955293 (89k:58271)

7. M. Braverman and V. Silantyev, Kirwan-Novikov inequalities on a manifold with boundary, Trans. Amer. Math. Soc. 358 (8) (2006), 3329-3361. MR2218978 (2007d:57049)

8. K. Chang and J. Liu, A cohomology complex for manifolds with boundary, Topol. Methods Nonlinear Anal. 5 (2) (1995), 325-340. MR1374068 (96k:58206)

9. J. Cheeger, On the Hodge theory of Riemannian pseudomanifolds, Geometry of the Laplace operator (Proc. Sympos. Pure Math., Univ. Hawaii, Honolulu, Hawaii, 1979), Proc. Sympos. Pure Math., XXXVI, 91-146, Amer. Math. Soc., Providence, R.I., 1980. MR.573430 (83a:58081)

10. A. Dold, Lectures on algebraic topology, Classics in Mathematics, Springer-Verlag, 1995. MR.1335915 (96c:55001)

11. M. Farber and E. Shustin, Witten deformation and polynomial differential forms, Geom. Dedicata 80 (1-3) (2000), 125-155. MR,1762505(2001i:58042)

12. M. Goresky and R. MacPherson, Intersection homology theory, Topology 19 (1980), 135-165. MR572580 (82b:57010)

13. M. Goresky and R. MacPherson, Stratified Morse theory, Ergebnisse der Mathematik und ihrer Grenzgebiete (3) [Results in Mathematics and Related Areas (3)] 14 (1988), SpringerVerlag. MR932724 (90d:57039)

14. B. Helffer, Semi-classical analysis for the Schrödinger operator and applications, Lecture Notes in Mathematics, 1336, Springer-Verlag, 1988. MR960278 (90c:81043)

15. B. Helffer, M. Klein and F. Nier, Quantitative analysis of metastability in reversible diffusion processes via a Witten complex approach, Mat. Contemp. 26 (2004), 41-85. MR2111815 (2005i:58025)

16. B. Helffer and F. Nier, Quantitative analysis of metastability in reversible diffusion processes via a Witten complex approach: the case with boundary, Mém. Soc. Math. Fr., Nouv. Sér. 105 (2006). MR2270650 (2007k:58044)

17. B. Helffer and J. Sjöstrand, Puits multiples en mécanique semi-classique. IV. Étude du complexe de Witten, Comm. Partial Differential Equations 10 (3) (1985), 245-340. MR780068 (87i:35162)

18. D. Le Peutrec, Local WKB construction for Witten Laplacians on manifolds with boundary, Analysis \& PDE 3 (3) (2010), 227-260. MR2672794 (2011j:58035)

19. U. Ludwig, A proof of the stratified Morse inequalities for singular complex curves using the Witten deformation, Ann. Inst. Fourier 61 (5) (2011), 1749-1777.

20. U. Ludwig, The Witten complex for singular spaces of dimension two with cone-like singularities, Math. Nachr. 284 (5-6) (2011), 717-738. MR.2663764

21. U. Ludwig, The Witten deformation for even dimensional spaces with cone-like singularities and admissible Morse functions, C. R., Math., Acad. Sci. Paris 348 (15-16) (2010) 915-918. MR2677991(2011i:58030) 
22. M. Reed and B. Simon, Methods of modern mathematical physics, New York, Academic Press, 1980. MR751959 (85e:46002)

23. E. Witten, Supersymmetry and Morse theory, J. Differential Geom. 17 (4) (1982), 661-692. MR683171 (84b:58111)

Mathematisches Institut, Universität Freiburg, Eckerstrasse 1, 79104 Freiburg, GerMANY

E-mail address: ursula.ludwig@math.uni-freiburg.de 